\title{
SUPERCONNECTIONS AND HIGHER INDEX THEORY
}

\author{
J. LoTT
}

\begin{abstract}
Let $M$ be a smooth closed spin manifold. The higher index theorem, as given for example in Proposition 6.3 of [CM], computes the pairing between the group cohomology of $\pi_{1}(M)$ and the Chern character of the "higher" index of a Dirac-type operator on M. Using superconnections, we give a heat equation proof of this theorem on the level of differential forms on a noncommutative base space. As a consequence, we obtain a new proof of the Novikov conjecture for hyperbolic groups.
\end{abstract}

\section{Introduction}

Let $M$ be a smooth closed connected spin manifold. Let $V$ be a Hermitian vector bundle on $M$. If $M$ is even-dimensional, the Atiyah-Singer index theorem identifies the topological expression $\int_{M} \widehat{A}(M) \wedge \mathrm{Ch}(V)$ with the index of the Dirac-type operator acting on $L^{2}$-sections of the bundle $S(M) \otimes$ $V$, where $S(M)$ is the spinor bundle on $M$ [AS1].

When $M$ is not simply-connected, one can refine the index theorem to take the fundamental group into account $[\mathrm{Co} 3, \mathrm{Kas}, \mathrm{Mo}]$. Let $\Gamma$ denote the fundamental group of $M$. Let $\nu: M \rightarrow B \Gamma$ be the classifying map for the universal cover $\widetilde{M}$ of $M$. For $[\eta] \in H^{*}(B \Gamma ; \mathbb{C})$, higher index theory attempts to identify $\int_{M} \widehat{A}(M) \wedge \mathrm{Ch}(V) \wedge \nu^{*}[\eta]$ with an analytic expression. The main topological and geometric applications of higher index theory are to Novikov's conjecture on homotopy-invariants of non-simply-connected manifolds [No], and to questions of the existence of positive-scalar-curvature metrics on $M[\mathrm{Ro}]$.

In order to motivate the statement of the higher index theorem, let us first recall how Lusztig used the index theorem for families of operators 
to prove a higher index theorem in the case of $\Gamma=\mathbf{Z}^{k}[\mathrm{Lu}]$. Let $T^{k}=$ $\operatorname{Hom}(\Gamma, U(1))$ be the dual group to $\Gamma$ and let $L_{\theta}$ be the flat unitary line bundle over $M$ whose holonomy is specified by $\theta \in T^{k}$. Consider the product fibration $M \rightarrow M \times T^{k} \rightarrow T^{k}$. Suppose for simplicity that $M$ is evendimensional; then there is a bundle $\mathcal{H}$ over $T^{k}$ of $\mathbf{Z}_{2}$-graded Hilbert spaces, where $\mathcal{H}_{\theta}$, the fiber over $\theta \in T^{k}$, consists of the $L^{2}$-sections of $S(M) \otimes V \otimes L_{\theta}$. There is also a family $Q$ of vertical Dirac-type operators parametrized by $T^{k}$, where $\mathrm{Q}_{\theta}$ acts on $\mathcal{H}_{\theta}$. The analytic index $\operatorname{Index}(\mathrm{Q})$ of the family of elliptic operators, as defined in [AS2], lies in $K^{0}\left(T^{k}\right)$. An element $[\eta]$ of the group cohomology $H^{\ell}\left(\mathbf{Z}^{k} ; \mathbb{C}\right)$ gives a homology class $\tau_{\eta} \in H_{\ell}\left(T^{k} ; \mathbb{C}\right)$, against which the Chern character $\mathrm{Ch}(\operatorname{Index}(\mathrm{Q})) \in H^{*}\left(T^{k} ; \mathbb{C}\right)$ can be paired. The families index theorem [AS2] then implies

$$
\int_{\tau_{\eta}} \operatorname{Ch}(\operatorname{Index}(\mathrm{Q}))=\text { const. }(l) \int_{M} \widehat{A}(M) \wedge \mathrm{Ch}(V) \wedge \nu^{*}[\eta],
$$

giving the desired analytic interpretation of the right-hand-side. The purpose of $[\mathrm{Lu}]$ was to apply $(*)$ to the Novikov conjecture.

In order to extend these methods to nonabelian $\Gamma$, let us note some algebraic properties of the above construction. The algebra of continuous functions $C\left(T^{k}\right)$ acts on the vector space $C(\mathcal{H})$ of continuous sections of $\mathcal{H}$ by multiplication. Upon performing Fourier transform over $T^{k}, C(\mathcal{H})$ maps to a certain subspace of the $L^{2}$-sections of the pullback bundle $S(\widetilde{M}) \otimes \widetilde{V}$ on $\widetilde{M}$, this subspace thus being a $C\left(T^{k}\right)$-Hilbert module in the sense of [Kas].

The generalization of Lùsztig's method to nonabelian $\Gamma$ is based on a "fibration" $M \rightarrow P \rightarrow B$ which exists only morally, where $B$ is a noncommutative space whose "algebra of continuous functions" is taken to be the algebra $\Lambda=C_{r}^{*} \Gamma$, the reduced group $C^{*}$-algebra [Co3]. (When $\Gamma=\mathbf{Z}^{k}$, $\Lambda \cong C\left(T^{k}\right)$.) Mishchenko and Kasparov define a Hilbert $\Lambda$-module of $L^{2}$ sections of $S(\widetilde{M}) \otimes \widetilde{V}$, upon which a Dirac-type operator $\widetilde{D}$ acts. The analytic index of $\widetilde{D}$ lies in " $K^{0}(B)$ ", or more precisely in $K_{0}(\Lambda)$ [Mi, Kas]. The Mishchenko-Fomenko index theorem identifies the analytic index with a topological index [MF].

In order to pair these indices with the group cohomology of $\Gamma$, one needs additional structure on $B$. Let $\mathfrak{B}^{\infty}$ be a dense subalgebra of $\Lambda$ containing $\mathrm{C} \Gamma$ which is stable under the holomorphic functional calculus of $\Lambda$ [Co1]. (For example, if $\Gamma=\mathbf{Z}^{k}$, one can take $\mathfrak{B}^{\infty}$ to be $C^{\infty}\left(T^{k}\right)$.) Then $K_{0}(\Lambda) \cong$ $K_{0}\left(\mathfrak{B}^{\infty}\right)$. One can think of the image of Index $(\widetilde{D})$ under this isomorphism as being a "smoothing" of Index $(\widetilde{D})$. 
One can then use the fact that $K_{0}\left(\mathfrak{B}^{\infty}\right)$ pairs with the cyclic cohomology $H C^{*}\left(\mathfrak{B}^{\infty}\right)$ of $\mathfrak{B}^{\infty}[\mathrm{Co} 1]$ to extract numbers from $\operatorname{Index}(\widetilde{D})$. In loose but more familiar terms, the Chern character $\mathrm{Ch}(\operatorname{Index}(\widetilde{D}))$ lies in the "cohomology" of $B$. More precisely, it lies in the cyclic homology group $H C_{*}\left(\mathfrak{B}^{\infty}\right)$ [Col, Ka]. One then wants to define a "homology class" of $B$ which one can pair with $\mathrm{Ch}(\operatorname{Index}(\tilde{D}))$. The correct notion of homology for $B$ is given by the (periodic) cyclic cohomology of $\mathfrak{B}^{\infty}$. In particular, given a group cocycle $\eta \in Z^{l}(\Gamma ; \mathbb{C})$, one obtains an cyclic cocycle $\tau_{\eta} \in Z C^{l}(\mathbb{C} \Gamma)$ (eqn. (62)). If $\tau_{\eta}$ extends to an element of $Z C^{l}\left(\mathfrak{B}^{\infty}\right)$ then Proposition 6.3 of $[\mathrm{CM}]$ gives

$$
\left\langle\operatorname{Ch}(\operatorname{Index}(\widetilde{D})), \tau_{\eta}\right\rangle=\text { const. }(l) \int_{M} \widehat{A}(M) \wedge \operatorname{Ch}(V) \wedge \nu^{*}[\eta] .
$$

The special case when $l=0$ is the $L^{2}$-index theorem [At].

An equivalent and more concrete description of the above "fibration" is given by a vector bundle $\mathcal{E}$ over $M$ whose fibers are finitely-generated right projective $\mathfrak{B}$-modules for an appropriate algebra $\mathfrak{B}[\mathrm{Mi}]$. We will use this latter description in making things precise, although we will move back and forth freely between the two pictures.

In another direction, using Quillen's theory of superconnections [Q], Bismut gave a heat equation proof of the Atiyah-Singer families index theorem on the level of differential forms on the base space [Bi]. Equation (*) is a consequence.

Analogously, we wish to give a heat equation proof of $(* *)$. Our original purpose was to study higher versions of spectral invariants, such as the eta invariant [Lo1]. These higher eta invariants should enter into a higher index theorem for manifolds with boundary. However, it turned out to be necessary to first understand the case of closed manifolds, i.e. equation (**), in terms of superconections. This is what we present here.

As in [Bi], we wish to produce an explicit differential form on $B$ which represents $\operatorname{Ch}(\operatorname{Index}(\widetilde{D}))$. First, one needs to know what a form on the noncommutative space $B$ should mean. A differential complex $\bar{\Omega}_{*}(\mathfrak{B})$ was defined in $[\mathrm{Ka}]$, and its homology can be identified with a subspace of the cyclic homology of the relevant algebra $\mathfrak{B}$. In Section II we briefly review this theory. In this section we also consider integral operators on sections of $\mathcal{E}$ and define their traces and supertraces.

In the case at hand, the relevant vector bundles $\mathcal{E}$ come from a flat $\mathfrak{B}$ bundle over $M$. There is some choice in exactly which subalgebra $\mathfrak{B}$ of $\Lambda$ is 
taken. In Section III we consider a subalgebra $\mathfrak{B}^{\omega}$ of $\Lambda$ consisting of elements whose coefficients decay faster than any exponential in a word-length metric. If $\Gamma=\mathbf{Z}$ then $\mathfrak{B}^{\boldsymbol{\omega}}$ is isomorphic to the restrictions of holomorphic functions on $\mathbb{C}-0$ to the unit circle, and so $\mathfrak{B}^{\omega}$ is like an algebra of "analytic" functions on $B$. (The technical reason for the appearance of this algebra is the existence of finite-propagation-speed estimates for heat kernels on $\widetilde{M}$.) The smooth sections $\Gamma^{\infty}\left(\mathcal{E}^{\omega}\right)$ of the corresponding vector bundle $\mathcal{E}^{\omega}$ are shown to correspond to smooth sections of $S(\widetilde{M}) \otimes \widetilde{V}$ with rapid decay. Using this description, we make the trace of Section II more explicit.

By construction, the vector space of smooth sections of $\mathcal{E}^{\omega}$ is a right $\mathfrak{B}^{\omega}$-module. Let $\nabla: \Gamma^{\infty}\left(\mathcal{E}^{\omega}\right) \rightarrow \Gamma^{\infty}\left(\mathcal{E}^{\omega} \otimes_{\mathfrak{B}^{\omega}} \Omega_{1}\left(\mathfrak{B}^{\omega}\right)\right)$ be a connection on $\mathcal{E}^{\omega}$. This is, in a sense, a connection in the vertical direction of $\mathcal{E}^{\omega}$, when thought of as a vector bundle over $M$. Let $Q$ be the Dirac-type operator on $\Gamma^{\infty}\left(\mathcal{E}^{\omega}\right)$. Applying Quillen's formalism [Q], for any $\beta, s>0$, the Chern character of $\mathcal{E}^{\omega}$ is defined to be

$$
\operatorname{ch}_{\beta, s}\left(\mathcal{E}^{\omega}\right)=\operatorname{STR} \exp \left(-\beta(\nabla+s Q)^{2}\right) \in \bar{\Omega}_{*}\left(\mathfrak{B}^{\omega}\right)
$$

To make this expression useful, one needs an explicit description of a connection on $\mathcal{E}^{\omega}$. In Section IV we show that the simplest such connection comes from a function $h \in C_{0}^{\infty}(\widetilde{M})$ with the property that the sum of the translates of $h$ is 1 . Then $(* * *)$ is a well-defined closed element of $\bar{\Omega}_{*}\left(\mathfrak{B}^{\omega}\right)$, and its homology class is independent of $s$.

Given a group cocycle $\eta^{*} \in Z^{l}(\Gamma ; \mathbb{C})$, if the corresponding cyclic cocycle $\tau_{\eta} \in Z C^{l}(\mathbb{C} \Gamma)$ extends to an element of $Z C^{l}\left(\mathfrak{B}^{\omega}\right)$ then the pairing

$$
\left\langle\operatorname{ch}_{\beta, s}\left(\mathcal{E}^{\omega}\right), \tau_{\eta}\right\rangle \in \mathbb{C}
$$

is well-defined and independent of $s$. As usual with heat equation approaches to index theory, the $s \rightarrow 0$ limit of $(* * * *)$ becomes the integral of a local expression on $M$. In Section $V$ we compute this limit. (The local analysis is easier than in [Bi], as there is no need to use a Levi-Civita superconnection.) The limit must involve $\nu^{*}[\eta]$, and it may seem strange that this could become a local expression on $M$, but this is where the choice of $h$ enters. In Proposition 12 we find

$$
\lim _{s \rightarrow 0}\left\langle\operatorname{ch}_{\beta, s}\left(\mathcal{E}^{\omega}\right), \tau_{\eta}\right\rangle=\beta^{l / 2} /(l !) \int_{M} \widehat{A}(M) \wedge \mathrm{Ch}(V) \wedge \omega
$$


where $\omega$ is a closed $l$-form on $M$ whose pullback to $\widetilde{M}$ is given by

$$
\pi^{*} \omega=\sum R_{g_{1}}^{*} d h \wedge \ldots \wedge R_{g_{l}}^{*} d h \eta\left(e, g_{1}, \ldots, g_{l}\right) \in \Lambda^{l}(\widetilde{M})
$$

We then show that $\omega$ represents $\nu^{*}[\eta] \in H^{l}(M ; \mathbb{C})$.

It remains to show that

$$
\left\langle\operatorname{ch}_{\beta, s}\left(\mathcal{E}^{\omega}\right), \tau_{\eta}\right\rangle=\left\langle\operatorname{Ch}_{\beta}(\operatorname{Index}(\widetilde{D})), \tau_{\eta}\right\rangle .
$$

For this, we find it necessary to work with the algebra $\mathfrak{B}^{\infty}$ and assume that $\tau_{\eta}$ extends to a cyclic cocycle of $\mathfrak{B}^{\infty}$. In Section VI we sketch a proof of $(* * * * *)$. We reduce to the case of invertible $\widetilde{D}$, and then use a trick of [Bi] to show the equality. This completes the proof of $(* *)$.

One application of $(* *)$ is to the Novikov conjecture. Taking $\widetilde{D}$ to be the signature operator, the right-hand-side of $(* *)$ becomes const. $(l) \int_{M} L(M) \wedge$ $\nu^{*}[\eta]$, where $L(M) \in H^{*}(M ; \mathbb{C})$ is the Hirzebruch $L$-polynomial. The Novikov conjecture states that this "higher" signature is an (orientationpreserving) homotopy invariant of $M$. One can show that $\operatorname{Index}(\widetilde{D}) \in K_{0}(\Lambda)$ is a homotopy invariant of $M[\mathrm{Mi}, \mathrm{Kas}, \mathrm{HS}]$. If the group $\Gamma$ is such that one can apply $(* *)$ then the validity of the Novikov conjecture follows. In particular, in [CM] it was shown that if $\Gamma$ is hyperbolic in the sense of Gromov [GH] then $(* *)$ applies. Thus our proof of $(* *)$ gives a new proof of the validity of the Novikov conjecture for hyperbolic groups. One can also apply (**) to find obstructions to the existence of positive-scalar-curvature metrics on $M$ [Ro]. If one takes $\widetilde{D}$ to be the pure Dirac operator then if $M$ has positive scalar curvature, $\operatorname{Index}(\widetilde{D})$ vanishes. Thus if the group $\Gamma$ is such that one can apply $(* *), \int_{M} \widehat{A}(M) \wedge \nu^{*}[\eta]$ is an obstruction to the existence of a positive-scalar-curvature metric on $\mathrm{M}$.

In [Lo1] a bivariant Chern character was proposed in the case of finitelygenerated projective modules. The obstacle to defining a bivariant Chern character for more general projective modules was the lack of a good trace theory for Hilbert modules. In the present case there is such a trace. The smooth sections of $\mathcal{E}^{\infty}=\mathcal{E}^{\omega} \otimes_{\mathfrak{B} \omega} \mathfrak{B}^{\infty}$ form a $\left(C^{\infty}(M), \mathfrak{B}^{\infty}\right)$-bivariant module, and the pairing $\left\langle\operatorname{ch}_{\beta, s}, \tau_{\eta}\right\rangle$ of the bivariant Chern character with $\tau_{\eta}$ is a cocycle in the space $C_{\epsilon}^{*}\left(C^{\infty}(M)\right)$ of entire cyclic cochains [Co2]. In Section VII we compute the $s \rightarrow 0$ limit of $\left\langle\operatorname{ch}_{\beta, s}, \tau_{\eta}\right\rangle$.

Heat equation methods were also used in the paper of Connes and Moscovici $[\mathrm{CM}]$ to attack the Novikov conjecture, and it is worth comparing 
the two approaches. One difference is that we use heat kernels to form the Chern character of a superconnection as in $(* * *)$, whereas in [CM] the heat kernels are used to form an idempotent matrix over an algebra of smoothing operators [CM, Section 2]. Theorem 5.4 of [CM] is similar to our Corollary 2, but is stronger in that it is a statement about $\mathbb{C} \Gamma$, whereas Corollary 2 is a statement about $\mathfrak{B}^{\omega}$. We believe that there is some point to taking a superconnection approach to these questions, as there should be interesting extensions.

This paper is an extension of [Lo1], in which the finite-dimensional analog was worked out. An exposition of the Mischenko-Fomenko theorem and related results appears in [Hi].

I wish to thank Dan Burghelea and Jeff Cheeger for useful suggestions, and Henri Moscovici for helpful discussions.

\section{Algebraic Preliminaries}

Let $\mathfrak{B}$ be a Fréchet locally $m$-convex algebra with unit, i.e. the projective limit of a sequence of Banach algebras with unit [Mal]. We first define a graded differential algebra $(\mathrm{GDA}) \widehat{\Omega}_{*}(\mathfrak{B})$. This will be an appropriate completion of

$$
\Omega_{*}(\mathfrak{B})=\bigoplus_{k=0}^{\infty} \Omega_{k}(\mathfrak{B}),
$$

the universal GDA of $\mathfrak{B}[\mathrm{Co} 1, \mathrm{Ka}]$. As a vector space, $\Omega_{k}(\mathfrak{B})$ is given by

$$
\Omega_{k}(\mathfrak{B})=\mathfrak{B} \otimes\left(\otimes^{k}(\mathfrak{B} / \mathbb{C})\right) .
$$

As a $\mathrm{GDA}, \Omega_{*}(\mathfrak{B})$ is generated by $\mathfrak{B}$ and $d \mathfrak{B}$ with the relations

$$
d 1=0, \quad d^{2}=0, \quad d\left(\omega_{k} \omega_{\ell}\right)=\left(d \omega_{k}\right) \omega_{\ell}+(-1)^{k} \omega_{k}\left(d \omega_{\ell}\right)
$$

for $\omega_{k} \in \Omega_{k}(\mathfrak{B}), \omega_{\ell} \in \Omega_{\ell}(\mathfrak{B})$. It will be convenient to write an element $\omega_{k}$ of $\Omega_{k}(\mathfrak{B})$ as a finite sum $\sum b_{0} d b_{1} \ldots d b_{k}$. Recall that the homology of the differential complex $\bar{\Omega}_{*}(\mathfrak{B})=\Omega_{*}(\mathfrak{B}) /\left[\Omega_{*}(\mathfrak{B}), \Omega_{*}(\mathfrak{B})\right]$ is isomorphic to a subspace of the reduced cyclic homology of $\mathfrak{B}[\mathrm{Ka}]$. (This statement must be modified in degree zero, for which we refer to [Ka].)

Let $\Theta_{*}(\mathfrak{B})$ denote the GDA

$$
\Theta_{*}(\mathfrak{B})=\bigoplus_{k=0}^{\infty}\left(\otimes^{k+1} \mathfrak{B}\right),
$$


with the product given by

$$
\left(b_{0} \otimes b_{1} \otimes \ldots \otimes b_{k}\right)\left(c_{0} \otimes c_{1} \otimes \ldots \otimes c_{\ell}\right)=b_{0} \otimes b_{1} \otimes \ldots \otimes b_{k} c_{0} \otimes c_{1} \otimes \ldots \otimes c_{\ell}
$$

and the differential given by

$$
\begin{aligned}
d\left(b_{0} \otimes b_{1} \otimes \ldots \otimes b_{k}\right)= & 1 \otimes b_{0} \otimes b_{1} \otimes \ldots \otimes b_{k}-b_{0} \otimes 1 \otimes b_{1} \otimes \ldots \\
& \otimes b_{k}+\ldots+(-1)^{k+1} b_{0} \otimes b_{1} \otimes \ldots \otimes b_{k} \otimes 1 .
\end{aligned}
$$

Give $\Theta_{k}(\mathfrak{B})$ the projective tensor product topology, with closure $\widehat{\Theta}_{k}(\mathfrak{B})$. Let

$$
\widehat{\Theta}_{*}(\mathfrak{B})=\prod_{k=0}^{\infty} \widehat{\Theta}_{k}(\mathfrak{B})
$$

denote the completion of $\Theta_{*}(\mathfrak{B})$ in the product topology.

Proposition 1. $\widehat{\Theta}_{*}(\mathfrak{B})$ is a Fréchet GDA.

There is a natural embedding $e$ of $\Omega_{*}(\mathfrak{B})$, as a graded differential algebra, in $\widehat{\Theta}_{*}(\mathfrak{B})$, with

$$
\mathfrak{e}(b)=b, \quad \mathfrak{e}(d b)=1 \otimes b-b \otimes 1
$$

Let $\widehat{\Omega}_{*}(\mathfrak{B})$ denote the closure of $\mathfrak{e}\left(\Omega_{*}(\mathfrak{B})\right)$ in $\widehat{\Theta}_{*}(\mathfrak{B})$.

Corollary 1. $\widehat{\Omega}_{*}(\mathfrak{B})$ is a Fréchet GDA.

Define $\overline{\widehat{\Omega}}_{*}(\mathfrak{B})$ to be $\widehat{\Omega}_{*}(\mathfrak{B}) / \overline{\left[\widehat{\Omega}_{*}(\mathfrak{B}), \widehat{\Omega}_{*}(\mathfrak{B})\right]}$. Let $\bar{H}_{*}(\mathfrak{B})$ denote the homology of the differential complex $\overline{\widehat{\Omega}}_{*}(\mathfrak{B})$.

Let $\mathfrak{E}$ be a Fréchet space which is a (continuous) right $\mathfrak{B}$-module. If $\mathfrak{F}$ is a Fréchet space which is a (continuous) left $\mathfrak{B}$-module, let $\mathfrak{E} \otimes \mathfrak{F}$ be the projective topological tensor product of $\mathfrak{E}$ and $\mathfrak{F}$. Let $\mathfrak{H}$ be the closure in $\mathfrak{E} \widehat{\otimes} \mathfrak{F}$ of

$$
\operatorname{span}\{e b \otimes f-e \otimes b f: e \in \mathfrak{E}, f \in \mathfrak{F}, b \in \mathfrak{B}\} .
$$

We put $\mathfrak{E} \widehat{\otimes}_{\mathfrak{B}} \mathfrak{F}$ to be the Fréchet space $(\mathfrak{E} \widehat{\otimes} \mathfrak{F}) / \mathfrak{H}$.

With this definition, $\mathfrak{E} \widehat{\otimes}_{\mathfrak{B}} \widehat{\Omega}_{k}(\mathfrak{B})$ is isomorphic to the closure of the algebraic tensor product $\mathfrak{E} \otimes_{\mathfrak{B}} \Omega_{k}(\mathfrak{B}) \subset \mathfrak{E} \otimes_{\mathfrak{B}}\left(\otimes^{k+1} \mathfrak{B}\right)=\mathfrak{E} \otimes\left(\otimes^{k} \mathfrak{B}\right)$ in $\mathfrak{E} \widehat{\otimes}\left(\widehat{\otimes}^{k}(\mathfrak{B})\right)$, where the latter has the projective tensor product topology. 
For the rest of this section, we assume that $\mathfrak{E}$ is a finitely generated right projective $\mathfrak{B}$-module. Let $\mathfrak{F}$ be a Fréchet $\mathfrak{B}$-bimodule. Then there is a trace

$$
\operatorname{Tr}: \operatorname{Hom}_{\mathfrak{B}}\left(\mathfrak{E}, \mathfrak{E} \widehat{\otimes}_{\mathfrak{B}} \mathfrak{F}\right) \rightarrow \mathfrak{F} / \overline{[\mathfrak{B}, \mathfrak{F}]} .
$$

To define $\operatorname{Tr}$, write $\mathfrak{E}$ as $e \mathfrak{B}^{n}$, with $e$ a projector in $M_{n}(\mathfrak{B})$. Then an operator $T \in \operatorname{Hom}_{\mathfrak{B}}\left(\mathfrak{E}, \mathfrak{E} \widehat{\otimes}_{\mathfrak{B}} \mathfrak{F}\right)=\operatorname{Hom}_{\mathfrak{B}}\left(e \mathfrak{B}^{n}, e \mathfrak{F}^{n}\right)$ can be represented by a matrix $T \in M_{n}(\mathfrak{F})$ satisfying $e T=T e=T$. Put

$$
\operatorname{Tr}(T)=\sum_{i=1}^{n} T_{i i} \quad(\bmod \overline{[\mathfrak{B}, \mathfrak{F}]}) .
$$

This is independent of the choices made. (We quotient by the closure of $[\mathfrak{B}, \mathfrak{F}]$ to ensure that the trace takes value in a Fréchet space.)

LEMMA 1. Suppose that $\mathfrak{E}$ and $\mathfrak{E}^{\prime}$ are finitely generated right projective $\mathfrak{B}$ modules and $\mathfrak{F}$ is a Fréchet algebra containing $\mathfrak{B}$. Given $T \in$ $\operatorname{Hom}_{\mathfrak{B}}\left(\mathfrak{E}, \mathfrak{E}^{\prime} \widehat{\otimes}_{\mathfrak{B}} \mathfrak{F}\right)$ and $T^{\prime} \in \operatorname{Hom}_{\mathfrak{B}}\left(\mathfrak{E}^{\prime}, \mathfrak{E} \widehat{\otimes}_{\mathfrak{B}} \mathfrak{F}\right)$, let $T^{\prime} T \in \operatorname{Hom}_{\mathfrak{B}}\left(\mathfrak{E}, \mathfrak{E} \widehat{\otimes}_{\mathfrak{B}} \mathfrak{F}\right)$ and $T T^{\prime} \in \operatorname{Hom}_{\mathfrak{B}}\left(\mathfrak{E}^{\prime}, \mathfrak{E}^{\prime} \widehat{\otimes}_{\mathfrak{B}} \mathfrak{F}\right)$ be the induced products. Then $\operatorname{Tr}\left(T^{\prime} T\right)=$ $\operatorname{Tr}\left(T T^{\prime}\right) \in \mathfrak{F} / \overline{[\mathfrak{F}, \mathfrak{F}]}$.

We omit the proof.

In the case that $\mathfrak{E}$ is $\mathbf{Z}_{2}$-graded by an operator $\Gamma_{\mathfrak{E}} \in$ End $_{\mathfrak{B}}(\mathfrak{E})$ satisfying $\Gamma_{\mathfrak{E}}^{2}=1$, we can extend the trace to a supertrace by $\operatorname{Tr}_{s}(T)=\operatorname{Tr}\left(\Gamma_{\mathfrak{E}} T\right)$.

Let $M$ be a closed connected oriented smooth Riemannian manifold. Let $\mathcal{E}$ be a smooth $\mathfrak{B}$-vector bundle on $M$ with fibers isomorphic to $\mathcal{E}$. This means that if $\mathcal{E}$ is defined using charts $\left\{U_{\alpha}\right\}$, then a transition function is a smooth map $\phi_{\alpha \beta}: U_{\alpha} \cap U_{\beta} \rightarrow$ End $_{\mathfrak{B}}(\mathcal{E})$. We will denote the fiber over $m \in M$ by $\mathcal{E}_{m}$. If $\mathfrak{F}$ is a Fréchet algebra containing $\mathfrak{B}$, let $\mathcal{E} \widehat{\otimes}_{\mathfrak{B}} \mathfrak{F}$ denote the $\mathfrak{B}$-vector bundle with fibers $\left(\mathcal{E} \widehat{\otimes}_{\mathfrak{B}} \mathfrak{F}\right)_{m}=\mathcal{E}_{m} \widehat{\otimes}_{\mathfrak{B}} \mathfrak{F}$ and transition functions $\phi_{\alpha \beta} \widehat{\otimes}_{\mathfrak{B}} \dot{I} d_{\mathfrak{F}} \in$ End $_{\mathfrak{B}}\left(\mathfrak{E} \widehat{\otimes}_{\mathfrak{B} F} \mathfrak{F}\right)$. Let $\Gamma^{\infty}(\mathcal{E})$ denote the right $\mathfrak{B}$-module of smooth sections of $\mathcal{E}$.

DEFINITION: Let $\operatorname{Hom}_{\mathfrak{B}}^{\infty}\left(\mathcal{E}, \mathcal{E} \widehat{\otimes}_{\mathfrak{B}} \mathfrak{F}\right)$ be the algebra of integral operators $T: \Gamma^{\infty}(\mathcal{E}) \rightarrow \Gamma^{\infty}\left(\mathcal{E} \widehat{\otimes}_{\mathfrak{B}} \mathfrak{F}\right)$ with smooth kernels $T\left(m_{1}, m_{2}\right) \quad \in$ $\operatorname{Hom}_{\mathfrak{B}}\left(\mathcal{E}_{m_{2}}, \mathcal{E}_{m_{1}} \widehat{\otimes}_{\mathfrak{B} \mathfrak{F})}\right.$. That is, for $s \in \Gamma^{\infty}(\mathcal{E})$,

$$
(T s)\left(m_{1}\right)=\int_{M} T\left(m_{1}, m_{2}\right) s\left(m_{2}\right) \operatorname{dvol}\left(m_{2}\right) \in \mathcal{E}_{m_{1}} \widehat{\otimes}_{\mathfrak{B}} \mathfrak{F}
$$


Definition: For $T \in \operatorname{Hom}_{\mathfrak{B}}^{\infty}\left(\mathcal{E}, \mathcal{E} \widehat{\otimes}_{\mathfrak{B}} \mathfrak{F}\right)$,

$$
\operatorname{TR}(T)=\int_{M} \operatorname{Tr}(T(m, m)) d \operatorname{vol}(m) \in \mathfrak{F} / \overline{[\mathfrak{F}, \mathfrak{F}]}
$$

PRoposition 2. TR is a trace.

Proof: We have

$$
\left(T T^{\prime}\right)\left(m, m^{\prime}\right)=\int_{M} T\left(m, m^{\prime \prime}\right) T^{\prime}\left(m^{\prime \prime}, m^{\prime}\right) \operatorname{dvol}\left(m^{\prime \prime}\right)
$$

Then

$$
\begin{aligned}
\operatorname{TR}\left(T T^{\prime}\right)= & \int_{M} \operatorname{Tr}\left(T\left(m, m^{\prime \prime}\right) T^{\prime}\left(m^{\prime \prime}, m\right)\right) \operatorname{dvol}\left(m^{\prime \prime}\right) d \operatorname{vol}(m)= \\
& \int_{M} \operatorname{Tr}\left(T^{\prime}\left(m^{\prime \prime}, m\right) T\left(m, m^{\prime \prime}\right)\right) \operatorname{dvol}(m) \operatorname{dvol}\left(m^{\prime \prime}\right)=\operatorname{TR}\left(T^{\prime} T\right)
\end{aligned}
$$

If the fibers of $\mathcal{E}$ are $\mathbf{Z}_{2}$-graded, we can extend TR to a supertrace STR on $\operatorname{Hom}_{\mathfrak{B}}^{\infty}\left(\mathcal{E}, \mathcal{E} \widehat{\otimes}_{\mathfrak{B}} \mathfrak{F}\right)$ by

$$
\operatorname{STR}(T)=\int_{M} \operatorname{Tr}_{s}(T(m, m)) d \operatorname{vol}(m) \in \mathfrak{F} / \overline{[\mathfrak{F}, \mathfrak{F}]}
$$

\section{III. $\mathfrak{B}^{\boldsymbol{\omega}}$-Bundles}

Let $\Gamma$ be a finitely-generated discrete group and let $\|\circ\|$ be a right-invariant word-length metric on $\Gamma$. For $q \in \mathbf{Z}$, define the Hilbert space

$$
\ell^{2, q}(\Gamma)=\left\{f: \Gamma \rightarrow \mathbb{C}:|f|_{q}^{2}=\sum_{g} \exp (2 q\|g\|)|f(g)|^{2}<\infty\right\}
$$

and let $\mathfrak{B}^{\omega}$ be the vector space

$$
\mathfrak{B}^{\omega}=\bigcap_{q} \ell^{2, q}(\Gamma)
$$

LEMMA 2.

$$
\mathfrak{B}^{\omega}=\left\{f: \Gamma \rightarrow \mathbf{C}: \text { for all } q \in \mathbf{Z}, \sup _{g}(\exp (q\|g\|)|f(g)|)<\infty\right\} .
$$


Proof: If $f \in \mathfrak{B}^{\omega}$ then for all $q \in \mathbf{Z}, \exp (2 q\|g\|)|f(g)|^{2}$ is bounded in $g$, and so $\exp (q\|g\|)|f(g)|$ is bounded in $g$. Suppose that $f: \Gamma \rightarrow \mathbb{C}$ is such that for all $r \in \mathbf{Z}$,

$$
\sup _{g}(\exp (r\|g\|)|(g)|)=C_{r}<\infty .
$$

Then $\sum_{g} \exp (2 q\|g\|)|f(g)|^{2} \leq C_{r}^{2} \sum_{g} \exp (2(q-r)\|g\|)$. As $\Gamma$ has at most exponential growth, by taking $r$ large enough we can ensure that the last sum is finite.

Proposition 3. $\mathfrak{B}^{\omega}$ is independent of the choice of $\|\circ\|$, and is an algebra with unit under convolution.

Proof: As all word-length metrics are quasi-isometric [GH], the independence follows. If $T \in \mathfrak{B}^{\omega}$ and $f \in \ell^{2, q}(\Gamma)$, we will show that

$$
|T * f|_{q} \leq \text { const. }(q, T)|f|_{q} .
$$

If we then take both $T$ and $f$ in $\mathfrak{B}^{\omega}$, the proposition will follow.

Let $f_{h}$ denote $f(h)$. Then

$$
\begin{aligned}
& \left|\sum_{h} T_{g h^{-1}} f_{h}\right|^{2} \leq \\
& \left(\sum_{h .} \exp (-q\|h\|)\left|T_{g h^{-1}}\right|^{1 / 2}\left|T_{g h^{-1}}\right|^{1 / 2} \exp (q\|h\|)\left|f_{h}\right|\right)^{2} \leq \\
& \left(\sum_{h} \exp (-2 q\|h\|)\left|T_{g h^{-1}}\right|\right)\left(\sum_{h^{\prime}}\left|T_{g h^{\prime-1}}\right| \exp \left(2 q\left\|h^{\prime}\right\|\right)\left|f_{h^{\prime}}\right|^{2}\right) .
\end{aligned}
$$

Thus

$$
\begin{aligned}
& |T * f|_{q}^{2}=\sum_{g} \exp (2 q\|g\|)\left|\sum_{h} T_{g h^{-1}} f_{h}\right|^{2} \leq \\
& \sum_{g}\left(\sum_{h} \exp (2 q(\|g\|-\|h\|))\left|T_{g h^{-1}}\right|\right)\left(\sum_{h^{\prime}}\left|T_{g h^{\prime}-1}\right| \exp \left(2 q\left\|h^{\prime}\right\|\right)\left|f_{h^{\prime}}\right|^{2}\right) \leq \\
& \sum_{g}\left(\sum_{h} \exp \left(2 q\left\|g h^{-1}\right\|\right)\left|T_{g h^{-1}}\right|\right)\left(\sum_{h^{\prime}}\left|T_{g h^{\prime-1}}\right| \exp \left(2 q\left\|h^{\prime}\right\|\right)\left|f_{h^{\prime}}\right|^{2}\right) \leq(21) \\
& \left(\sum_{k} \exp (2 q\|k\|)\left|T_{k}\right|\right)\left(\sum_{\ell}\left|T_{\ell}\right|\right)\left(\sum_{h^{\prime}} \exp \left(2 q\left\|h^{\prime}\right\|\right)\left|f_{h^{\prime}}\right|^{2}\right)= \\
& \left(\sum_{k} \exp (2 q\|k\|)\left|T_{k}\right|\right)\left(\sum_{\ell}\left|T_{\ell}\right|\right)|f|_{q}^{2} .
\end{aligned}
$$


Let $\Lambda$ denote the reduced group $C^{*}$-algebra of $\Gamma$, namely the completion of $\mathbb{C} \Gamma$ with respect to the operator norm on $B\left(\ell^{2}(\Gamma)\right)$, where $\mathbb{C} \Gamma$ acts on $\ell^{2}(\Gamma)$ by convolution.

There is a Fréchet topology on $\mathfrak{B}^{\omega}$ coming from its definition as a projective limit of Hilbert spaces. There is also a description of $\mathfrak{B}^{\boldsymbol{\omega}}$ as a Fréchet locally $m$-convex algebra. Namely, put

$$
\begin{gathered}
\mathcal{P}=\{T \in \Lambda: \text { for all } q \in \mathbf{Z}, T \text { acts as a bounded operator by } \\
\text { convolution on } \left.\ell^{2, q}(\Gamma)\right\} .
\end{gathered}
$$

By its definition, $\mathcal{P}$ is equipped with a sequence of norms.

Proposition 4. As topological vector spaces, $\mathfrak{B}^{\boldsymbol{\omega}}=\mathcal{P}$.

Proof: By the proof of Proposition $3, \mathfrak{B}^{\omega}$ injects continuously into $\mathcal{P}$. Applying an element $T$ of $\mathcal{P}$ to the element $e \in \bigcap_{q} \ell^{2, q}(\Gamma)$ gives a continous injection of $\mathcal{P}$ into $\mathfrak{B}^{\omega}$. These two maps are clearly inverses of each other.

It follows that $\mathfrak{B}^{\omega}$ has a holomorphic functional calculus.

Note. $\mathfrak{B}^{\omega}$ is generally not holomorphically closed in $\Lambda$. For example, if $\Gamma=\mathbf{Z}$ then an element $T$ of $\mathfrak{B}^{\omega}$ can be identified with its Fourier transform $T=\sum T_{g} z^{g}$, a holomorphic function on $\mathbb{C}-0$. This identification gives $\mathfrak{B}^{\omega} \cong H(\mathbb{C}-0)$. On the other hand, in this case $\Lambda \cong C\left(S^{1}\right)$. Taking for example $T=z \in H(\mathbb{C}-0) \subset C\left(S^{1}\right)$, the spectrum of $T$ in $C\left(S^{1}\right)$ consists of the unit circle. If $f$ is the holomorphic function defined on a neighborhood of the unit circle by $f(w)=(w-2)^{-1}, f(T)$ is well-defined in $C\left(S^{1}\right)$, but does not lie in $H(\mathbb{C}-0)$.

Let $\Gamma$ denote the fundamental group of $M$. Let $\widetilde{M}$ denote the universal cover of $M$, on which $g \in \Gamma$ acts on the right by $R_{g} \in \operatorname{Diff}(\widetilde{M})$. Denote the covering map by $\pi: \widetilde{M} \rightarrow M$. As $\Gamma$ acts on $\mathfrak{B}^{\omega}$ on the left, we can form $\widetilde{M} \times_{\Gamma} \mathfrak{B}^{\omega}$, a flat $\mathfrak{B}^{\omega}$-bundle over $M$. Let $E$ be a Hermitian vector bundle with Hermitian connection on $M$ and let $\widetilde{E}$ be the pullback of $E$ to $\widetilde{M}$, with the pulled-back connection. Let $R_{g}^{*} \in \operatorname{Aut}(\widetilde{E})$ denote the action of $g \in \Gamma$ on $\widetilde{E}$.

Definition: $\mathcal{E}^{\omega}=\left(\widetilde{M} \times_{\Gamma} \mathfrak{B}^{\omega}\right) \otimes E$, a $\mathfrak{B}^{\omega}$-bundle over $M$.

Fix a base point $x_{0} \in \widetilde{M}$. 
Proposition 5. There is an isomorphism $L: \Gamma^{\infty}\left(\mathcal{E}^{\omega}\right) \rightarrow\left\{f \in C^{\infty}(\widetilde{M}, \widetilde{E}):\right.$ for all $q \in \mathbf{Z}$ and all multi-indices $\alpha$,

$$
\left.\sup _{x}\left(\exp \left(q d\left(x_{0}, x\right)\right)\left|\nabla^{\alpha} f(x)\right|\right)<\infty\right\} .
$$

Proof: By the construction of $\mathcal{E}^{\omega}, \Gamma^{\infty}\left(\mathcal{E}^{\omega}\right)$ consists of the $\Gamma$-equivariant elements of $C^{\infty}\left(\widetilde{M}, \widetilde{E} \otimes \mathfrak{B}^{\omega}\right)$. Writing $s \in \Gamma^{\infty}\left(\mathcal{E}^{\omega}\right)$ as $\sum_{g} s_{g} g$ with $s_{g} \in$ $C^{\infty}(\widetilde{M}, \widetilde{E})$, the equivariance means that

$$
R_{\gamma}^{*} \gamma s=s \text { for all } \gamma \in \Gamma .
$$

This becomes $\sum_{g}\left(R_{\gamma}^{*} s_{g}\right) \gamma g=\sum_{g} s_{\gamma g} \gamma g$, and so $R_{\gamma}^{*} s_{g}=s_{\gamma g}$ for all $\gamma, g \in \Gamma$. Thus $s_{g}=R_{g}^{*} s_{1}$, and so $s=\sum_{g}\left(R_{g}^{*} s_{1}\right) g$.

Let $L$ be the map which takes $s$ to $s_{1}$. We will show that $L$ is the desired isomorphism. First, if $\widetilde{m} \in \widetilde{M}$ then

$$
s(\tilde{m})=\sum_{g}\left(R_{g}^{*} s_{1}\right)(\tilde{m}) g \in \widetilde{E}_{\tilde{m}} \otimes \mathfrak{B}^{\omega} .
$$

Thus for all $q \in \mathbf{Z}, \sup _{g}\left(\exp (q\|g\|)\left|s_{1}(\tilde{m} g)\right|\right)<\infty$. By the smoothness of $s$, we have such an estimate uniformly for $\widetilde{m}$ lying within a fundamental domain of $\widetilde{M}$ containing $x_{0}$. As $\widetilde{M}$ is quasi-isometric to $\Gamma$ [GH], there are constants $A>0$ and $B \geq 0$ such that for all $x \in \widetilde{M}$ and $g \in \Gamma$,

$$
A^{-1}\|g\|-B \leq d\left(x g^{-1}, x\right) \leq A\|g\|+\mathrm{B} .
$$

Then

$$
\begin{aligned}
& \exp \left(q d\left(x_{0}, x\right)\right)\left|s_{1}(x)\right| \leq \\
& \exp \left(q d\left(x_{0}, x g^{-1}\right)\right) \exp \left(q d\left(x g^{-1}, x\right)\right)\left|s_{1}\left(x g^{-1} g\right)\right| \leq \\
& \text { const. } \exp \left(q d\left(x_{0}, x g^{-1}\right)\right) \exp (q A\|g\|)\left|s_{1}\left(x g^{-1} g\right)\right| .
\end{aligned}
$$

By choosing $g$ so that $x g^{-1}$ lies within a fundamental domain containing $x_{0}$, we obtain from (26) that $\exp \left(q d\left(x_{0}, x\right)\right)\left|s_{1}(x)\right|$ is uniformly bounded in $x$. The same argument applies to the covariant derivatives of $s_{1}$.

Now suppose that $f \in C^{\infty}(\widetilde{M}, \widetilde{E})$ is such that for all $q \in \mathbf{Z}$ and all multi-indices $\alpha$,

$$
\sup _{x}\left(\exp \left(q d\left(x_{0}, x\right)\right)\left|\nabla^{\alpha} f(x)\right|\right)<\infty
$$


Put $L^{\prime}(f)=\sum_{g}\left(R_{g}^{*} f\right) g$. We must show that $L^{\prime}(f) \in \Gamma^{\infty}\left(\mathcal{E}^{\omega}\right)$. It will then follow that $L^{\prime}$ is an inverse to $L$.

By construction, $L^{\prime}(f)$ is $\Gamma$-equivariant. Let $\left\{V_{\alpha}\right\}$ be a collection of charts on $M$ over which $E$ is trivialized. Then we can reduce to the case that $E$ is a trivial $\mathbb{C}$-bundle and $f \in C^{\infty}\left(V_{\alpha} \times \Gamma, \mathbb{C}\right)$, with the above decay conditions. It is enough to show that when restricted to $V_{\alpha} \times\{e\}, \Sigma_{g}\left(R_{g}^{*} f\right) g$ represents a smooth map from $V_{\alpha}$ to $\mathfrak{B}^{\omega}$. For $\widetilde{m} \in V_{\alpha} \times\{e\}$,

$$
\left(\sum\left(R_{g}^{*} f\right) g\right)(\tilde{m})=\sum f(\tilde{m} g) g
$$

and so for all $q \in \mathbf{Z}$,

$$
\begin{aligned}
& \exp (q\|g\|)|f(\tilde{m} g)| \leq \\
& \text { const. } \exp (q A d(\widetilde{m}, \widetilde{m} g))|f(\widetilde{m} g)| \leq \\
& \text { const. } \exp \left(q A d\left(\widetilde{m}, x_{0}\right)\right) \exp \left(q A d\left(x_{0}, \tilde{m} g\right)\right)|f(\widetilde{m} g)| \leq \\
& \text { const. } \sup _{x}\left(\exp \left(q A d\left(x_{0}, x\right)\right)|f(x)|\right)<\infty .
\end{aligned}
$$

Thus $\sum_{g}\left(R_{g}^{*} f\right) g$ is a map from $V_{\alpha}$ to $\mathfrak{B}^{\omega}$. Doing the same estimates using covariant derivatives gives the smoothness.

Proposition 6. The algebra End $\mathfrak{B}^{\omega}\left(\mathcal{E}^{\omega}\right) \equiv \operatorname{Hom}_{\mathfrak{B}^{\omega}}^{\infty}\left(\mathcal{E}^{\omega}, \mathcal{E}^{\omega}\right)$ is isomorphic to the algebra of $\Gamma$-invariant integral operators $T$ on $L^{2}(\widetilde{M}, \widetilde{E})$ with smooth kernels $T(x, y) \in \operatorname{Hom}\left(\widetilde{E}_{y}, \widetilde{E}_{x}\right)$ such that for all $q \in \mathbf{Z}$ and multi-indices $\alpha$ and $\beta$,

$$
\sup _{x, y}\left(\exp (q d(x, y))\left|\nabla_{x}^{\alpha} \nabla_{y}^{\beta} T(x, y)\right|\right)<\infty
$$

We omit the proof, which is similar to that of Proposition 5.

Let $\phi \in C_{0}^{\infty}(\widetilde{M})$ be such that

$$
\sum_{g} R_{g}^{*} \phi=1
$$

Let tr denote the local trace on $\operatorname{End}\left(\widetilde{E}_{x}\right)$. 
Note. We now have defined three traces: $\operatorname{tr}$ is the trace on $\operatorname{End}\left(\widetilde{E}_{x}\right), \operatorname{Tr}$ is the trace on End ${ }_{\mathfrak{B}^{\omega}}\left(\mathcal{E}_{m}^{\omega}\right)$ and TR is the trace on End $\mathfrak{B}^{\infty}\left(\mathcal{E}^{\omega}\right)$. If $E$ is $\mathbf{Z}_{2}$-graded, the corresponding supertraces are denoted $\operatorname{tr}_{s}, \operatorname{Tr}_{s}$ and STR.

Proposition 7. Representing an element $T \in$ End $_{\mathfrak{B}^{\omega}}^{\infty}\left(\mathcal{E}^{\omega}\right)$ by an operator $\widetilde{T} \in B\left(L^{2}(\widetilde{M}, \widetilde{E})\right)$ as in Proposition 6, its trace is given by

$$
\begin{aligned}
\operatorname{TR}(T) & =\sum_{g}\left[\int_{\widetilde{M}} \phi(x) \operatorname{tr}\left(\left(R_{g}^{*} \tilde{T}\right)(x, x)\right) d \operatorname{vol}(x)\right] g \quad\left(\bmod \overline{\left[\mathfrak{B}^{\omega}, \mathfrak{B}^{\omega}\right]}\right) \\
& =\sum_{g}\left[\int_{\widetilde{M}} \phi(x) \operatorname{tr}(\widetilde{T}(x g, x)) \operatorname{dvol}(x)\right] g \quad\left(\bmod \overline{\left[\mathfrak{B}^{\omega}, \mathfrak{B}^{\omega}\right]}\right) .
\end{aligned}
$$

Proof: The proof is a matter of unraveling the isomorphisms of Propositions 5 and 6 . Let $\left\{V_{\alpha}\right\}$ be a collection of charts on $M$ over which $E$ is trivialized. Then we can reduce to the case that $E$ is a trivial $\mathbb{C}$-bundle. We have $\pi^{-1}\left(V_{\alpha}\right) \cong V_{\alpha} \times \Gamma$. For $\widetilde{m}_{1}, \widetilde{m}_{2} \in V_{\alpha} \times\{e\}$, we can use isomorphisms to represent

$$
T\left(m_{1}, m_{2}\right) \in \operatorname{Hom}_{\mathfrak{B}^{\omega}}^{\infty}\left(\mathcal{E}_{m_{2}}^{\omega}, \mathcal{E}_{m_{1}}^{\omega}\right) \cong \operatorname{Hom}_{\mathfrak{B}^{\omega}}\left(\mathfrak{B}^{\omega}, \mathfrak{B}^{\omega}\right) \cong \mathfrak{B}^{\omega}
$$

by $\sum_{g} \widetilde{T}\left(\tilde{m}_{1} g, \tilde{m}_{2}\right) g$. Then

$$
\begin{aligned}
& \int_{V_{\alpha}} \operatorname{Tr}(T(m, m)) d \operatorname{vol}(m)= \\
& \int_{V_{\alpha}} \sum_{g} \tilde{T}(m g, m) g d v o l(m) \quad\left(\bmod \overline{\left[\mathfrak{B}^{\omega}, \mathfrak{B}^{\omega}\right]}\right)= \\
& \int_{V_{\alpha}} \sum_{g} \sum_{\gamma} \phi(m \gamma) \tilde{T}(\operatorname{mg} \gamma, m \gamma) g \operatorname{dvol}(m) \quad\left(\bmod \overline{\left[\mathfrak{B}^{\omega}, \mathfrak{B}^{\omega}\right]}\right)= \\
& \int_{V_{\alpha}} \sum_{g} \sum_{\gamma} \phi(m \gamma) \widetilde{T}\left(m \gamma \gamma^{-1} g \gamma, m \gamma\right) g d \operatorname{vol}(m) \quad\left(\bmod \overline{\left[\mathfrak{B}^{\omega}, \mathfrak{B}^{\omega}\right]}\right)= \\
& \int_{V_{\alpha}} \sum_{g} \sum_{\gamma} \phi(m \gamma) \tilde{T}(m \gamma g, m \gamma) \gamma g \gamma^{-1} d \operatorname{vol}(m) \quad\left(\bmod \overline{\left[\mathfrak{B}^{\omega}, \mathfrak{B}^{\omega}\right]}\right)= \\
& \int_{V_{\alpha}} \sum_{g} \sum_{\gamma} \phi(m \gamma) \tilde{T}(m \gamma g, m \gamma)\left(g+\left[\gamma g, \gamma^{-1}\right]\right) d v o l(m)\left(\bmod \overline{\left[\mathfrak{B}^{\omega}, \mathfrak{B}^{\omega}\right]}\right)= \\
& \int_{V_{\alpha}} \sum_{\gamma} \sum_{g} \phi(m \gamma) \tilde{T}(m \gamma g, m \gamma) g d v o l(m) \quad\left(\bmod \overline{\left[\mathfrak{B}^{\omega}, \mathfrak{B}^{\omega}\right]}\right)=
\end{aligned}
$$




$$
\int_{\pi^{-1}\left(V_{\alpha}\right)} \sum_{g} \phi(x) \widetilde{T}(x g, x) g \operatorname{dvol}(x) \quad\left(\bmod \overline{\left[\mathfrak{B}^{\omega}, \mathfrak{B}^{\omega}\right]}\right) .
$$

Using a partition of unity subordinate to $\left\{V_{\alpha}\right\}$ and adding the contributions of the various charts gives (31).

We now give the extension of the previous propositions to form-valued sections of $\mathcal{E}^{\omega}$. With the notation of Section II, put $\mathfrak{F}^{\omega}=\widehat{\Omega}_{*}\left(\mathfrak{B}^{\omega}\right)$. As in Proposition 5, we can represent an element $f$ of $\Gamma^{\infty}\left(\mathcal{E}^{\omega} \widehat{\otimes}_{\mathfrak{B} \omega} \mathfrak{F}^{\omega}\right)$ of degree $k$ as $\sum f_{g_{1} \ldots g_{k}} d g_{1} \ldots d g_{k}$, with each $f_{g_{1} \ldots g_{k}} \in C^{\infty}(\widetilde{M}, \widetilde{E})$ a smooth rapidly decreasing section of $\widetilde{E}$. As in Proposition 6, we can represent an element $K$ of $\operatorname{Hom}_{\mathfrak{B}^{\omega}}^{\infty}\left(\mathcal{E}^{\omega}, \mathcal{E}^{\omega} \widehat{\otimes}_{\mathfrak{B} \omega} \mathfrak{F}^{\omega}\right)$ of degree $k$ by smooth rapidly decreasing kernels $K_{g_{1} \ldots g_{k}}(x, y) \in \operatorname{Hom}\left(\widetilde{E}_{y}, \widetilde{E}_{x}\right)$ such that $K=\sum K_{g_{1} \ldots g_{k}} d g_{1} \ldots d g_{k}$ is $\Gamma$-invariant. Then for $f \in \Gamma^{\infty}\left(\mathcal{E}^{\omega}\right)$ we have

$$
(K f)(x)=\sum \int_{\widetilde{M}} K_{g_{1} \ldots g_{k}}(x, y) f(y) d v o l(y) d g_{1} \ldots d g_{k}
$$

As in Proposition 7, we have

$$
\operatorname{TR}(K)=\sum \int_{\widetilde{M}} \phi(x) \operatorname{tr}\left(K_{g_{1} \ldots g_{k}}\left(x g_{0}, x\right)\right) d v o l(x) g_{0} d g_{1} \ldots d g_{k}
$$

\section{The Chern Character}

Now suppose in addition that $M^{n}$ is even-dimensional and spin. Let $S$ be the $\mathbf{Z}_{2}$-graded spinor bundle on $M$, with the Levi-Civita connection, and let $V$ be a Hermitian bundle on $M$ with Hermitian connection. Take $E$ to be $S \otimes V$. Let $Q$ denote the self-adjoint extension of the Dirac-type operator acting on $C_{0}^{\infty}(\widetilde{M}, \widetilde{E})[A t]$. In terms of a local framing of the tangent bundle,

$$
Q=-i \sum_{\mu=1}^{n} \gamma^{\mu} D_{\mu},
$$

with the Dirac matrices $\left\{\gamma^{\mu}\right\}_{\mu=1}^{n}$ satisfying

$$
\gamma^{\mu} \gamma^{\nu}+\gamma^{\nu} \gamma^{\mu}=2 \delta^{\mu \nu}
$$

Proposition 8. For $T>0, e^{-T Q^{2}} \in \operatorname{End}_{\mathfrak{B}^{\omega}}^{\infty}\left(\mathcal{E}^{\omega}\right)$. 
Proof: First, $e^{-T Q^{2}}$ is a $\Gamma$-invariant operator. By elliptic regularity, $e^{-T Q^{2}}(x, y)$ is smooth. Put $N=[n / 4]+1$. Let $\epsilon$ be a fixed sufficiently small number. If $d(x, y)>\epsilon$, put $R=d(x, y)-\epsilon$. By the finite-propagation-speed estimates of [CGT], we have the estimate [Lo2]

$$
\begin{aligned}
& \left|\left(Q^{2 k} e^{-T Q^{2}} Q^{2 \ell}\right)(x, y)\right| \leq \\
& \text { const. }\left(R^{2} / T\right)^{-1 / 2}\left[R^{-2(k+\ell)}+R^{-2(k+\ell)-4 N}+\right. \\
& \left.R^{2(k+\ell)} T^{-2(k+\ell)}+R^{2(k+\ell)+4 N} T^{-2(k+\ell)-4 N}\right] e^{-R^{2} / 4 T} .
\end{aligned}
$$

The requisite bounds on the covariant derivatives of $e^{-T Q^{2}}(x, y)$ follow by standard methods. Then the proposition follows from Proposition 6.

Note. In the "fibration" picture, the fact that $e^{-T Q^{2}}$ commutes with $\mathfrak{B}^{\omega}$ means that it corresponds to a family of vertical operators.

Let $h \in C_{0}^{\infty}(\widetilde{M})$ be such that

$$
\sum_{g} R_{g}^{*} h=1
$$

Given $f \in \Gamma^{\infty}\left(\mathcal{E}^{\omega}\right)$, considering it as an element of $C^{\infty}(\widetilde{M}, \widetilde{E})$ by Proposition 5 , define its covariant derivative to be

$$
\nabla_{g} f=h R_{g}^{*} f \in C^{\infty}(\widetilde{M}, \widetilde{E})
$$

Note that $C^{\infty}(M)$ acts on sections of $\Gamma^{\infty}\left(\mathcal{E}^{\omega} \widehat{\otimes}_{\mathfrak{B} \omega} \mathfrak{F}^{\omega}\right)$ by multiplication. PROPOSITION 9.

$$
\nabla f=\sum_{g} \nabla_{g} f \widehat{\otimes}_{\mathcal{B} \omega} d g
$$

defines a connection

$$
\nabla: \Gamma^{\infty}\left(\mathcal{E}^{\omega}\right) \rightarrow \Gamma^{\infty}\left(\mathcal{E}^{\omega} \widehat{\otimes}_{\mathfrak{B}^{\omega}} \widehat{\Omega}_{1}\left(\mathfrak{B}^{\omega}\right)\right)
$$

which commutes with the action of $C^{\infty}(M)$. 
Proof: We first show that $\nabla$ formally commutes with the action of $C^{\infty}(M)$. Given $\alpha \in C^{\infty}(M) ; \alpha$ acts on $C^{\infty}(\widetilde{M}, \widetilde{E})$ by multiplication by $\pi^{*}(\alpha)$. Then

$$
\begin{gathered}
\nabla(\alpha \cdot f)=\nabla\left(\pi^{*}(\alpha) f\right)=\sum_{g} h R_{g}^{*}\left(\pi^{*}(\alpha) f\right) \widehat{\otimes}_{\mathfrak{B}^{\omega}} d g= \\
\sum_{g} h \pi^{*}(\alpha) R_{g}^{*} f \widehat{\otimes}_{\mathfrak{B}^{\omega}} d g=\alpha \cdot \nabla f
\end{gathered}
$$

Thus $\nabla$ acts fiberwise on the vector bundle $\mathcal{E}^{\omega}$. To make this explicit, as in the proof of Proposition 5 we can consider the element $s$ of $\Gamma^{\infty}\left(\mathcal{E}^{\omega}\right)$ corresponding to $f$ to be a sum $s=\sum s_{g} g$, where $s_{g} \in C^{\infty}(\widetilde{M}, \widetilde{E})$ and $s_{g}=R_{g}^{*} f$. Then $\nabla s$ becomes

$$
\sum_{g, k} R_{g}^{*}\left(h R_{k}^{*} f\right) g d k=\sum_{g, k} R_{g}^{*} h\left(R_{g k}^{*} f\right) g d k=\sum_{g, k} R_{g}^{*} h s_{g k} g d k
$$

Applied to a point $\widetilde{m} \in \widetilde{M}$, we have

$$
\nabla\left(\sum_{g} s_{g}(\tilde{m}) g\right)=\sum_{g, k} h(\tilde{m} g) s_{g k}(\tilde{m}) g d k .
$$

Let

$$
\nabla_{m}: \mathcal{E}_{m}^{\omega} \rightarrow \mathcal{E}_{m}^{\omega} \widehat{\otimes}_{\mathfrak{B} \omega} \widehat{\Omega}_{1}\left(\mathfrak{B}^{\omega}\right)
$$

be the restriction of $\nabla$ to the fiber $\mathcal{E}_{m}^{\omega} \cong E_{m} \otimes \mathfrak{B}^{\omega}$ over $m=\pi(\tilde{m})$. Then $\nabla_{m}$ can be represented by

$$
\nabla_{m}\left(\sum_{g} t_{g} g\right)=\sum_{g, k} h(\tilde{m} g) t_{g k} g d k
$$

where $t_{g} \in \widetilde{E}_{\widetilde{m}}$.

By hypothesis, $t=\sum_{g} t_{g} g \in \mathcal{E}_{m}^{\omega} \cong E_{m} \otimes \mathfrak{B}^{\omega}$. We must show that $\nabla_{m}(t)$ is in $\mathcal{E}_{m}^{\omega} \widehat{\otimes}_{\mathfrak{B}^{\omega}} \widehat{\Omega}_{1}\left(\mathfrak{B}^{\omega}\right) \cong E_{m} \otimes \widehat{\Omega}_{1}\left(\mathfrak{B}^{\omega}\right)$.

As in Section II, let us think of $E_{m} \otimes \widehat{\Omega}_{1}\left(\mathfrak{B}^{\omega}\right)$ as embedded in $E_{m} \otimes$ $\mathfrak{B}^{\omega} \widehat{\otimes} \mathfrak{B}^{\omega}$. Then $\nabla_{m}(t)$ is formally represented as

$$
\nabla_{m}(t)=\sum_{g, k} h(\widetilde{m} g) t_{g k} g(1 \otimes k-k \otimes 1)=
$$




$$
\begin{aligned}
& \sum_{g, k} h(\tilde{m} g) t_{g k} g \otimes k-\sum_{g, k} h(\tilde{m} g) t_{g k} g k \otimes 1= \\
& \sum_{g, k} h(\tilde{m} g) t_{g k} g \otimes k-\sum_{g, k} h(\tilde{m} g) t_{k} k \otimes 1= \\
& \left(\sum_{g} h(\tilde{m} g) g \otimes \sum_{k} t_{g k} k\right)-t \otimes 1= \\
& \left(\sum_{g} h(\tilde{m} g) g \otimes\left(g^{-1} t\right)\right)-t \otimes 1 .
\end{aligned}
$$

As $h$ has compact support, the $g$-sum in $\sum_{g} h(\widetilde{m} g) g \otimes\left(g^{-1} t\right)$ is finite, and it follows that (48) makes sense in $E_{m} \otimes \mathfrak{B}^{\omega} \widehat{\otimes} \mathfrak{B}^{\omega}$.

We now show that $\nabla_{m}$ is a connection. If $\gamma \in \Gamma$,

$$
\begin{aligned}
& \nabla_{m}(t \gamma)=\nabla_{m}\left(\sum_{g} t_{g} g \gamma\right)=\nabla_{m}\left(\sum_{g} t_{g \gamma^{-1}} g\right)= \\
& \sum_{g, k} h(\tilde{m} g) t_{g k \gamma^{-1}} g d k=\sum_{g, k} h(\widetilde{m} g) t_{g k} g d(k \gamma)= \\
& \sum_{g, k} h(\tilde{m} g) t_{g k} g(d k) \gamma+\sum_{g, k} h(\tilde{m} g) t_{g k} g k d \gamma= \\
& \nabla_{m}(t) \gamma+\sum_{g, k} h(\tilde{m} g) t_{k} k d \gamma=\nabla_{m}(t) \gamma+t d \gamma .
\end{aligned}
$$

Then

$$
\nabla_{m}(t b)=\left(\nabla_{m} t\right) b+t \widehat{\otimes}_{\mathfrak{B} \omega} d b
$$

for any $b \in \mathfrak{B}^{\omega}$.

As $h$ is smooth, it follows that $\nabla$ is also a connection.

Note. There is a strong relationship between the connections $\nabla$ considered here and the partially flat connections of [Ka, Chapitre 4].

Define the superconnection

$$
D_{s}=\nabla+s Q \in \operatorname{Hom}^{\infty}\left(\mathcal{E}^{\omega}, \mathcal{E}^{\omega} \widehat{\otimes}_{\mathfrak{B}^{\omega}} \widehat{\Omega}_{*}\left(\mathfrak{B}^{\omega}\right)\right) .
$$

Then $D_{s}^{2} \in \operatorname{Hom}_{\mathfrak{B}^{\omega}}^{\infty}\left(\mathcal{E}^{\omega}, \mathcal{E}^{\omega} \widehat{\otimes}_{\mathfrak{B}^{\omega}} \widehat{\Omega}_{*}\left(\mathfrak{B}^{\omega}\right)\right)$ is given by

$$
D_{s}^{2}=s^{2} Q^{2}+s(\nabla Q+Q \nabla)+\nabla^{2} \text {. }
$$


Here $\nabla Q+Q \nabla$ is given explicitly by

$$
(\nabla Q+Q \nabla)(f)=\sum_{g}(\partial h) R_{g}^{*} f \widehat{\otimes}_{\mathfrak{B} \omega} d g
$$

where $f \in C^{\infty}(\widetilde{M}, \widetilde{E})$ and

$$
\partial h=[Q, h]=-i \sum_{\mu} \gamma^{\mu} \partial_{\mu} h
$$

and $\nabla^{2}$ is given by

$$
\nabla^{2}(f)=\sum_{g} \sum_{g^{\prime}} h R_{g}^{*} h R_{g g^{\prime}}^{*} f \widehat{\otimes}_{\mathfrak{B}^{\omega}} d g d g^{\prime} .
$$

Put

$$
\mathfrak{P}=-\left(s(\nabla Q+Q \nabla)+\nabla^{2}\right)
$$

and for $\beta>0$ define

$$
\exp \left(-\beta D_{s}^{2}\right) \in \operatorname{Hom}_{\mathfrak{B}^{\omega}}^{\infty}\left(\mathcal{E}^{\omega}, \mathcal{E}^{\omega} \widehat{\otimes}_{\mathfrak{B}^{\omega}} \widehat{\Omega}_{*}\left(\mathfrak{B}^{\omega}\right)\right)
$$

to be

$$
\begin{aligned}
\exp \left(-\beta D_{s}^{2}\right)= & \exp \left(-\beta s^{2} Q^{2}\right)+\int_{0}^{\beta} \exp \left(-u_{1} s^{2} Q^{2}\right) \mathfrak{P} \\
& \exp \left(-\left(\beta-u_{1}\right) s^{2} Q^{2}\right) d u_{1}+ \\
& \int_{0}^{\beta} \int_{0}^{u_{1}} \exp \left(-u_{1} s^{2} Q^{2}\right) \mathfrak{P} \exp a\left(-u_{2} s^{2} Q^{2}\right) \mathfrak{P} \\
& \exp \left(-\left(\beta-u_{1}-u_{2}\right) s^{2} Q^{2}\right) d u_{2} d u_{1}+\ldots
\end{aligned}
$$

As only a finite number of terms of the expansion of (58) contribute to the degree- $k$ component of $\exp \left(-\beta D_{s}^{2}\right)$, it is clear that (58) converges.

Definition: For $s>0$, the Chern character $\operatorname{ch}_{\beta, s}\left(\mathcal{E}^{\omega}\right) \in \overline{\widehat{\Omega}}_{\text {even }}\left(\mathfrak{B}^{\omega}\right)$ is given by

$$
\operatorname{ch}_{\beta, s}\left(\mathcal{E}^{\omega}\right)=\operatorname{STR} \exp \left(-\beta D_{s}^{2}\right) .
$$

Proposition 10. $\operatorname{ch}_{\beta, s}\left(\mathcal{E}^{\omega}\right)$ is closed.

We omit the proof, which is straightforward.

Proposition 11. The class of $\operatorname{ch}_{\beta, s}\left(\mathcal{E}^{\omega}\right)$ in $\bar{H}_{*}\left(\mathfrak{B}^{\omega}\right)$ is independent of $s \in$ $(0, \infty)$. 
Proof: Formally,

$$
\frac{d}{d s} \operatorname{ch}_{\beta, s}\left(\mathcal{E}^{\omega}\right)=d\left(-\beta \operatorname{STR} Q e^{-\beta D_{s}^{2}}\right)
$$

It is straightforward to check that this equation is valid. Then if $s_{1}, s_{2} \in$ $(0, \infty)$,

$$
\operatorname{ch}_{\beta, s_{1}}\left(\mathcal{E}^{\omega}\right)-\operatorname{ch}_{\beta, s_{2}}\left(\mathcal{E}^{\omega}\right)=d\left(-\beta \int_{s_{2}}^{s_{1}} \operatorname{STR} Q e^{-\beta D_{s}^{2}} d s\right)
$$

Let $\eta$ be an antisymmetric left-invariant (unnormalized) group $k$-cocycle. Then $\eta$ defines a cyclic $k$-cocycle $\tau_{\eta}$ on $\mathrm{C} \Gamma$ by

$$
\begin{aligned}
& \tau_{\eta}\left(g_{0}, \ldots, g_{k}\right)=\eta\left(g_{0}, g_{0} g_{1}, g_{0} g_{1} g_{2}, \ldots, g_{0} g_{1} \ldots g_{k}\right) \text { if } g_{0} g_{1} \ldots g_{k}=e \\
& \tau_{\eta}\left(g_{0}, \ldots, g_{k}\right)=0 \text { if } g_{0} g_{1} \ldots g_{k} \neq e \quad[\mathrm{Co} 1]
\end{aligned}
$$

Suppose that there are constants $C$ and $D$ so that

$$
\left|\tau_{\eta}\left(g_{0}, \ldots, g_{k}\right)\right| \leq C \exp \left(D\left(\left\|g_{0}\right\|+\ldots+\left\|g_{k}\right\|\right)\right)
$$

Then $\tau_{\eta}$ extends to a $k$-cocycle on $\mathfrak{B}^{\omega}$ and so can be paired with $\operatorname{ch}_{\beta, s}$. By Proposition 11, the pairing $\left\langle\operatorname{ch}_{\beta, s}\left(\mathcal{E}^{\omega}\right), \tau_{\eta}\right\rangle$ is independent of $s$.

\section{Small-Time Limit}

Proposition 12.

$$
\lim _{s \rightarrow 0}\left\langle\operatorname{ch}_{\beta, s}\left(\mathcal{E}^{\omega}\right), \tau_{\eta}\right\rangle=\beta^{k / 2} /(k !) \int_{M} \widehat{A}(M) \wedge \operatorname{Ch}(V) \wedge \omega
$$

where $\omega$ is the closed $k$-form on $M$ given by

$$
\pi^{*} \omega=\sum R_{g_{1}}^{*} d h \wedge \ldots \wedge R_{g_{k}}^{*} d h \eta\left(e, g_{1}, \ldots, g_{k}\right) \in \Lambda^{k}(\widetilde{M})
$$


Proof: First, let us consider the contribution to $\left\langle\operatorname{ch}_{\beta, s}, \tau_{\eta}\right\rangle$ coming from the term

$$
\begin{aligned}
& (-1)^{k} \int_{0}^{\beta} \ldots \int_{0}^{u_{k-1}} \exp \left(-u_{1} s^{2} Q^{2}\right) s(\nabla Q+Q \nabla) \exp \left(-u_{2} s^{2} Q^{2}\right) \\
& s(\nabla Q+Q \nabla) \ldots s(\nabla Q+Q \nabla) \exp \left(-\left(\beta-u_{1}-\ldots-u_{k}\right) s^{2} Q^{2}\right) d u_{k} \ldots d u_{1}
\end{aligned}
$$

of $\exp \left(-\beta D_{s}^{2}\right)$. Written out explicitly, this will be

$$
\begin{aligned}
& \sum(-1)^{k} \int_{0}^{\beta} \ldots \int_{0}^{u_{k-1}} \int_{\widetilde{M}} \phi\left(x_{0}\right) \operatorname{tr}_{s}\left[R_{g_{0}}^{*} \exp \left(-u_{1} s^{2} Q^{2}\right) s(\partial h) R_{g_{1}}^{*}\right. \\
& \exp \left(-u_{2} s^{2} Q^{2}\right) s(\partial h) R_{g_{2}}^{*} \ldots s(\partial h) R_{g_{k}}^{*} \\
& \left.\exp \left(-\left(\beta-u_{1}-\ldots-u_{k}\right) s^{2} Q^{2}\right)\right]\left(x_{0}, x_{0}\right) \\
& d v o l\left(x_{0}\right) d u_{k} \ldots d u_{1} \tau_{\eta}\left(g_{0}, \ldots, g_{k}\right)= \\
& \sum(-1)^{k} \int_{0}^{\beta} \ldots \int_{0}^{u_{k-1}} \int_{\widetilde{M}} \phi\left(x_{0}\right) \operatorname{tr}_{s}\left[\exp \left(-u_{1} s^{2} Q^{2}\right) s R_{g_{0}}^{*}(\partial h)\right. \\
& \quad \exp \left(-u_{2} s^{2} Q^{2}\right) s R_{g_{0} g_{1}}^{*}(\partial h) \ldots s R_{g_{0} \ldots g_{k-1}}^{*}(\partial h) \\
& \exp \left(-\left(\beta-u_{1}-\ldots-u_{k}\right) s^{2} Q^{2}\right) \\
& \left.R_{g_{0} \ldots g_{k}}^{*}\right]\left(x_{0}, x_{0}\right) d v o l\left(x_{0}\right) d u_{k} \ldots d u_{1} \tau_{\eta}\left(g_{0}, \ldots, g_{k}\right)=
\end{aligned}
$$

$$
\begin{aligned}
& \sum(-1)^{k} \int_{0}^{\beta} \ldots \int_{0}^{u_{k-1}} \int_{\widetilde{M}} \ldots \int_{\widetilde{M}} \phi\left(x_{0}\right) \operatorname{tr}_{s}\left[\exp \left(-u_{1} s^{2} Q^{2}\right)\left(x_{0}, x_{1}\right)\right. \\
& s(\partial h)\left(x_{1} g_{0}\right) \exp \left(-u_{2} s^{2} Q^{2}\right)\left(x_{1}, x_{2}\right) s(\partial h)\left(x_{2} g_{0} g_{1}\right) \ldots s(\partial h)\left(x_{k} g_{0} g_{1} \ldots g_{k-1}\right) \\
& \left.\exp \left(-\left(\beta-u_{1}-\ldots-u_{k}\right) s^{2} Q^{2}\right)\left(x_{k} g_{0} g_{1} \ldots g_{k}, x_{0}\right)\right] d v o l\left(x_{k}\right) \ldots d v o l\left(x_{0}\right) \\
& d u_{k} \ldots d u_{1} \tau_{\eta}\left(g_{0}, \ldots, g_{k}\right) .
\end{aligned}
$$

Because for small $s$ the heat kernels are concentrated near the diagonal, the only terms which will survive in the $s \rightarrow 0$ limit will have $g_{0} \ldots g_{k}=e$. Furthermore, the $s \rightarrow 0$ limit reduces to a question of local asymptotics on $\widetilde{M}$. By the Getzler calculus [G], (69) equals $(2 \pi)^{-n} \int_{T} \tilde{M} \operatorname{tr}_{s}(\sigma P)_{s^{-1}} d x d \xi$, where $P$ denotes the operator appearing in (69), $\sigma P$ is its symbol in the Getzler calculus and $(\sigma P)_{s^{-1}}$ is the rescaled symbol. A straightforward 
calculation gives that in the limit $s \rightarrow 0$, this becomes

$$
\begin{aligned}
& \sum(-1)^{k} \beta^{-k / 2}\left(\int_{0}^{\beta} \ldots \int_{0}^{u_{k-1}} d u_{k} \ldots d u_{1}\right) \int_{\widetilde{M}} \phi(x) \widehat{A}(x) \wedge \operatorname{Ch}(\widetilde{V})(x) \wedge \\
& d h\left(x g_{0}\right) \wedge \ldots \wedge d h\left(x g_{0} \ldots g_{k-1}\right) \eta\left(g_{0}, g_{0} g_{1}, g_{0} g_{1} g_{2}, \ldots, g_{0} g_{1} \ldots g_{k-1}, e\right)= \\
& \sum(-1)^{k} \beta^{k / 2} /(k !) \int_{\widetilde{M}} \phi \widehat{A}(\widetilde{M}) \wedge \operatorname{Ch}(\widetilde{V}) \wedge R_{g_{0}}^{*} d h \wedge \ldots \wedge R_{g_{0} \ldots g_{k-1}}^{*} d h \\
& \quad \eta\left(g_{0}, g_{0} g_{1}, g_{0} g_{1} g_{2}, \ldots, g_{0} \ldots g_{k-1}, e\right)= \\
& \quad \beta^{k / 2} /(k !) \int_{\widetilde{M}} \phi \widehat{A}(\widetilde{M}) \wedge \operatorname{Ch}(\widetilde{V}) \wedge \widetilde{\omega}
\end{aligned}
$$

where $\widetilde{\omega} \in \Lambda^{k}(\widetilde{M})$ is given by

$$
\tilde{\omega}=\sum R_{g_{1}}^{*} d h \wedge \ldots \wedge R_{g_{k}}^{*} d h \eta\left(e, g_{1}, \ldots, g_{k}\right) .
$$

Now let us consider the contribution to $\left\langle\mathrm{ch}_{\beta, s}, \tau_{\eta}\right\rangle$ coming from a term of $\exp \left(-\beta D_{s}^{2}\right)$ which contains a $\nabla^{2}$, such as, for example,

$$
\begin{aligned}
& (-1)^{k} \int_{0}^{\beta} \cdots \int_{0}^{u_{k-1}} \exp \left(-u_{1} s^{2} Q^{2}\right) \nabla^{2} \exp \left(-u_{2} s^{2} Q^{2}\right) s(\nabla Q+Q \nabla) \ldots \\
& s(\nabla Q+Q \nabla) \exp \left(-\left(\beta-u_{1}-\ldots-u_{k}\right) s^{2} Q^{2}\right) d u_{k} \ldots d u_{1} .
\end{aligned}
$$

Written out explicitly, this gives

$$
\begin{aligned}
\sum & (-1)^{k} \int_{0}^{\beta} \ldots \int_{0}^{u_{k-1}} \int_{\widetilde{M}} \ldots \int_{\widetilde{M}} \phi\left(x_{0}\right) \operatorname{tr}_{s}\left[\exp \left(-u_{1} s^{2} Q^{2}\right)\left(x_{0}, x_{1}\right)\right. \\
& h\left(x_{1} g_{0}\right) h\left(x_{1} g_{0} g_{1}\right) \exp \left(-u_{2} s^{2} Q^{2}\right)\left(x_{1}, x_{2}\right) s(\partial h)\left(x_{2} g_{0} g_{1} g_{1}^{\prime}\right) \ldots \\
& s(\partial h)\left(x_{k} g_{0} g_{1} g_{1}^{\prime} g_{2} \ldots g_{k-1}\right) \exp \left(-\left(\beta-u_{1}-\ldots-u_{k}\right) s^{2} Q^{2}\right) \\
& \left.\left(x_{k} g_{0} g_{1} g_{1}^{\prime} g_{2} \ldots g_{k}, x_{0}\right)\right] d \operatorname{vol}\left(x_{k}\right) \ldots \\
& d \operatorname{vol}\left(x_{0}\right) d u_{k} \ldots d u_{1} \tau_{\eta}\left(g_{0}, g_{1}, g_{1}^{\prime}, g_{2}, \ldots, g_{k}\right)
\end{aligned}
$$

By the Getzler calculus, in the $s \rightarrow 0$ limit, (75) becomes

$$
\begin{aligned}
& \sum(-1)^{k} \beta^{-(k-1) / 2}\left(\int_{0}^{\beta} \ldots \int_{0}^{u_{k-1}} d u_{k} \ldots d u_{1}\right) \int_{\widetilde{M}} \phi(x) \widehat{A}(x) \operatorname{Ch}(\widetilde{V})(x) \\
& \quad h\left(x g_{0}\right) h\left(x g_{0} g_{1}\right) d h\left(x g_{0} g_{1} g_{1}^{\prime}\right) \wedge \ldots \wedge d h\left(x g_{0} g_{1} g_{1}^{\prime} g_{2} \ldots g_{k-1}\right) \\
& \quad \eta\left(g_{0}, g_{0} g_{1}, g_{0} g_{1} g_{1}^{\prime}, \ldots, g_{0} g_{1} g_{1}^{\prime} g_{2} \ldots g_{k-1}, e\right)=
\end{aligned}
$$




$$
\begin{aligned}
& \sum(-1)^{k} \beta^{(k+1) / 2} /(k !) \\
& \quad \int_{\widetilde{M}} \phi \widehat{A}(\widetilde{M}) \wedge \operatorname{Ch}(\tilde{V}) \wedge R_{g_{0}}^{*} h \wedge R_{g_{0} g_{1}}^{*} h \wedge R_{g_{0} g_{1} g_{1}^{\prime}}^{*} d h \wedge \ldots \\
& \quad \wedge R_{g_{0} g_{1} g_{1}^{\prime} g_{2} \ldots g_{k-1}} d h \eta\left(g_{0}, g_{0} g_{1}, g_{0} g_{1} g_{1}^{\prime}, \ldots, g_{0} g_{1} g_{1}^{\prime} g_{2} \ldots g_{k-1}, e\right)= \\
& \quad \pm \beta^{(k+1) / 2} /(k !) \int_{\widetilde{M}} \phi \widehat{A}(\widetilde{M}) \wedge \mathrm{Ch}(\widetilde{V}) \wedge \widetilde{\omega}^{\prime}
\end{aligned}
$$

where $\widetilde{\omega}^{\prime} \in \Lambda^{k}(\widetilde{M})$ is given by

$$
\widetilde{\omega}^{\prime}=\sum R_{g_{1}}^{*} h \wedge R_{g_{1}^{\prime}}^{*} h \wedge \ldots \wedge R_{g_{k}}^{*} d h \eta\left(e, g_{1}, g_{1}^{\prime}, g_{2}, \ldots, g_{k}\right) .
$$

As $\eta\left(e, g_{1}, g_{1}^{\prime}, g_{2}, \ldots, g_{k}\right)$ is antisymmetric in $g_{1}$ and $g_{1}^{\prime}$, it follows that $\widetilde{\omega}^{\prime}$ vanishes. The same argument shows that all of the terms involving $\nabla^{2}$ vanish.

LEMMA 3. The form $\widetilde{\omega}$ of $(73)$ is a closed $\Gamma$-invariant form on $\widetilde{M}$.

Proof: $\widetilde{\omega}$ is clearly closed. For all $\gamma \in \Gamma$, we have

$$
\begin{aligned}
R_{\gamma}^{*} \widetilde{\omega}= & \sum R_{\gamma g_{1}}^{*} d h \wedge \ldots \wedge R_{\gamma g_{k}}^{*} d h \eta\left(e, g_{1}, \ldots, g_{k}\right)= \\
& \sum R_{g_{1}}^{*} d h \wedge \ldots \wedge R_{g_{k}}^{*} d h \eta\left(e, \gamma^{-1} g_{1}, \ldots, \gamma^{-1} g_{k}\right)= \\
& \sum R_{g_{1}}^{*} d h \wedge \ldots \wedge R_{g_{k}}^{*} d h \eta\left(\gamma, g_{1}, \ldots, g_{k}\right) .
\end{aligned}
$$

From the cocycle condition, this equals

$$
\begin{gathered}
\sum R_{g_{1}}^{*} d h \wedge \ldots \wedge R_{g_{k}}^{*} d h\left[\eta\left(e, g_{1}, \ldots, g_{k}\right)-\eta\left(e, \gamma, g_{2}, \ldots, g_{k}\right)+\ldots+\right. \\
\left.(-1)^{k} \eta\left(e, \gamma, g_{1}, \ldots, g_{k-1}\right)\right] .
\end{gathered}
$$

But for all $r$,

$$
\begin{aligned}
& \sum R_{g_{1}}^{*} d h \wedge \ldots \wedge R_{g_{k}}^{*} d h \eta\left(e, \gamma, g_{1}, \ldots, \widehat{g}_{r}, \ldots, g_{k}\right)= \\
& \pm\left(\sum_{g_{r}} R_{g_{r}}^{*} d h\right) \wedge \sum R_{g_{1}}^{*} d h \wedge \ldots \wedge R_{g_{r-1}}^{*} d h \wedge R_{g_{r+1}}^{*} d h \wedge \\
& \ldots \wedge R_{g_{k}}^{*} d h \eta\left(e, \gamma, g_{1}, \ldots, \widehat{g}_{r}, \ldots, g_{k}\right)
\end{aligned}
$$

and

$$
\sum_{g_{r}} R_{g_{r}}^{*} d h=d\left(\sum_{g_{r}} R_{g_{r}}^{*} h\right)=d(1)=0
$$


Thus only the first term of (81) contributes, and so

$$
R_{\gamma}^{*} \widetilde{\omega}=\sum R_{g_{1}}^{*} d h \wedge \ldots \wedge R_{g_{k}}^{*} d h \eta\left(e, g_{1}, \ldots, g_{k}\right)=\widetilde{\omega}
$$

End of Proof of Proposition 12: From Lemma 3, there is a closed form $\omega$ on $M$ such that $\widetilde{\omega}=\pi^{*}(\omega)$. Then

$$
\int_{\widetilde{M}} \phi \widehat{A}(\widetilde{M}) \wedge \operatorname{Ch}(\widetilde{V}) \wedge \widetilde{\omega}=\int_{M} \widehat{A}(M) \wedge \operatorname{Ch}(V) \wedge \omega
$$

We now wish to show that the cohomology class of the closed form $\tilde{\omega}$ is the pullback to $M$ of the cohomology class $[\eta]$ on $B \Gamma$. To do so, it is convenient to first relax the smoothness conditions on $\widetilde{\omega}$.

Let $h$ be a Lipschitz function on $\widetilde{M}$ of compact support with

$$
\sum_{g} R_{g}^{*} h=1 .
$$

As the distributional derivatives of a Lipschitz function are $L^{\infty}$-functions, it makes sense to define $\widetilde{\omega}_{h}$ by

$$
\widetilde{\omega}_{h}=\sum R_{g_{1}}^{*} d h \wedge \ldots \wedge R_{g_{k}}^{*} d h \eta\left(e, g_{1}, \ldots, g_{k}\right)
$$

a closed $\Gamma$-invariant $L^{\infty} k$-form on $\widetilde{M}$, and let $\omega_{h} \in \Lambda^{k}(M)$ be such that $\pi^{*} \omega_{h}=\widetilde{\omega}_{h}$. It is known that one can compute the de Rham cohomology of $M$ using flat forms (i.e. $L^{\infty}$-forms $\tau$ such that $d \tau$ is also $L^{\infty}$ ) [Te].

LEMMA 4. The cohomology class of $\omega_{h}$ is independent of $h$.

Proof: Let $h^{\prime}$ be another choice for $h$. Then

$$
\begin{gathered}
\widetilde{\omega}_{h}-\widetilde{\omega}_{h^{\prime}}=\sum\left[R_{g_{1}}^{*} d\left(h-h^{\prime}\right) \wedge \ldots \wedge R_{g_{k}}^{*} d h+\ldots+\right. \\
\left.R_{g_{1}}^{*} d h^{\prime} \wedge \ldots \wedge R_{g_{k}}^{*} d\left(h-h^{\prime}\right)\right] \eta\left(e, g_{1}, \ldots, g_{k}\right) .
\end{gathered}
$$

Put

$$
\tilde{\sigma}_{r}=\sum R_{g_{1}}^{*} d h^{\prime} \wedge \ldots R_{g_{r}}^{*}\left(h-h^{\prime}\right) \wedge \ldots \wedge R_{g_{k}}^{*} d h \eta\left(e, g_{1}, \ldots, g_{k}\right)
$$


a flat $(k-1)$-form on $\widetilde{M}$. Then

$$
\widetilde{\omega}_{h}-\widetilde{\omega}_{h^{\prime}}=d\left(\sum_{r=1}^{k}(-1)^{r+1} \widetilde{\sigma}_{r}\right)
$$

Furthermore, for all $\gamma \in \Gamma$,

$$
\begin{aligned}
R_{\gamma}^{*} \tilde{\sigma}_{r}= & \sum R_{g_{1}}^{*} d h^{\prime} \wedge \ldots R_{g_{r}}^{*}\left(h-h^{\prime}\right) \wedge \ldots \wedge R_{g_{k}}^{*} d h \eta\left(\gamma, g_{1}, \ldots, g_{k}\right)= \\
& \sum R_{g_{1}}^{*} d h^{\prime} \wedge \ldots R_{g_{r}}^{*}\left(h-h^{\prime}\right) \wedge \ldots \wedge R_{g_{k}}^{*} d h\left[\eta\left(e, g_{1}, \ldots, g_{k}\right)-\right. \\
& \left.\eta\left(e, \gamma, g_{2}, \ldots, g_{k}\right)+\ldots+(-1)^{k} \eta\left(e, \gamma, g_{1}, \ldots, g_{k-1}\right)\right] .
\end{aligned}
$$

As

$$
\sum_{g} R_{g}^{*} d h=\sum_{g} R_{g}^{*} d h^{\prime}=\sum_{g} R_{g}^{*}\left(h-h^{\prime}\right)=0
$$

it follows that $\widetilde{\sigma}_{r}$ is $\Gamma$-invariant. Then $\omega-\omega^{\prime}=d \sigma$, where $\sigma \in \Lambda^{k-1}(M)$ is such that

$$
\pi^{*} \sigma=\sum_{r=1}^{k}(-1)^{r+1} \widetilde{\sigma}_{r} .
$$

Let $X$ be the simplicial complex whose ordered cochain complex is the standard complex of $\Gamma[\mathrm{Br}]$. The $k$-simplices of $X$ are $(k+1)$-tuples of distinct elements of $\Gamma$. We will take $\Gamma$ to act on the right on $X$. Then the simplicial complex $X / \Gamma$ is a model for $B \Gamma$. For a vertex $v$, let $b_{v}$ denote the barycentric coordinate (on a simplex containing $v$ ) corresponding to $v$. Let $j$ be the continuous piecewise linear function on $X$ given by

$$
\begin{aligned}
j(x)= & 0 \text { if } x \in\left[g_{0}, \ldots, g_{k}\right] \text { and } g_{0} \neq e, \ldots, g_{k} \neq e \\
& b_{e} \text { if } x \in\left[g_{0}, \ldots, g_{k}\right] \text { and } g_{i}=e \text { for some } i
\end{aligned}
$$

Lemma $5 . \quad \sum_{g} R_{g}^{*} j=1$ 
Proof: Suppose that $x \in\left[g_{0}, \ldots, g_{k}\right]$. Then

$$
\sum_{g}\left(R_{g}^{*} j\right)(x)=\sum_{g} j(x g)=\sum_{i=0}^{k} j\left(x g_{i}^{-1}\right)=\sum_{i=0}^{k} b_{e}\left(x g_{i}^{-1}\right)=\sum_{i=0}^{k} b_{g_{i}}(x)=1 .
$$

Let $\widetilde{\omega}_{j}$ be the polynomial form on $X$, with coefficients in $\mathbb{C}$, given by

$$
\widetilde{\omega}_{j}=\sum R_{g_{1}}^{*} d j \wedge \ldots \wedge R_{g_{k}}^{*} d j \eta\left(e, g_{1}, \ldots, g_{k}\right) .
$$

Let $\omega_{j}$ be the polynomial form on $X / \Gamma$ such that $\widetilde{\omega}_{j}=\pi^{*} \omega_{j}$.

We define a $k$-cocycle $\tilde{\eta} \in C^{k}(X ; \mathbb{C})$ by putting

$$
\left\langle\widetilde{\eta},\left[\gamma_{0}, \gamma_{1}, \ldots, \gamma_{k}\right]\right\rangle=\eta\left(\gamma_{0}^{-1}, \ldots, \gamma_{k}^{-1}\right)
$$

By the left invariance of the group cocycle $\eta, \tilde{\eta}$ is right-invariant on $X$. With abuse of notation, let $\eta$ denote the corresponding simplicial cocycle on $X / \Gamma$. Proposition 13. As elements of $H^{k}(X / \Gamma ; \mathbb{C}),\left[\omega_{j}\right]=[\eta]$.

Proof: Let $A$ denote the de Rham map from polynomial forms on $X$ to $C^{*}(X)$. Then

$$
\begin{aligned}
& \left(A \omega_{j}\right)\left[\gamma_{0}, \ldots, \gamma_{k}\right]= \\
& \sum \eta\left(e, g_{1}, \ldots, g_{k}\right)\left\langle R_{g_{1}}^{*} d j \wedge \ldots \wedge R_{g_{k}}^{*} d j,\left[\gamma_{0}, \ldots, \gamma_{k}\right]\right\rangle= \\
& \sum \eta\left(e, \gamma_{i_{1}}^{-1}, \ldots, \gamma_{i_{k}}^{-1}\right)\left\langle R_{{\gamma_{i_{1}}}^{-1}}{ }^{*} d j \wedge \ldots \wedge R_{{\gamma_{i_{k}}}^{-1}}{ }^{*} d j,\left[\gamma_{0}, \ldots, \gamma_{k}\right]\right\rangle,
\end{aligned}
$$

where $i_{1}, \ldots, i_{k} \in\{0,1, \ldots, k\}$. Now (98) equals

$$
\sum \eta\left(e, \gamma_{i_{1}}^{-1}, \ldots, \gamma_{i_{k}}^{-1}\right)\left\langle d b_{\gamma_{i_{1}}} \wedge \ldots \wedge d b_{{\gamma_{k}}_{i_{k}}},\left[\gamma_{0}, \ldots, \gamma_{k}\right]\right\rangle \text {. }
$$

A simple calculation gives that (99) in turn equals

$$
\sum_{r=0}^{k}(-1)^{r+1} \eta\left(e, \gamma_{0}^{-1}, \ldots,{\widehat{\gamma_{r}}}^{-1}, \ldots, \gamma_{k}^{-1}\right)=\eta\left({\gamma_{0}^{-1}}^{-1} \ldots, \gamma_{k}^{-1}\right) \text {. }
$$

Thus $A\left(\omega_{j}\right)$ is the cochain $\eta$. As the de Rham map is an isomorphism on complex cohomology [GM], the proposition follows.

Let $\nu$ be the canonical (up to homotopy) map $\nu: M \rightarrow B \Gamma$ classifying the universal cover $\widetilde{M}$, with lift $\widetilde{\nu}: \widetilde{M} \rightarrow E \Gamma$.

Proposition 14. As elements of $H^{*}(M, \mathbb{C}),[\omega]=\nu^{*}([\eta])$. 
Proof: Let us triangulate $M$. Upon subdivision, we can homotop $\nu$ to be a simplicial map. Then with $h=\widetilde{\nu}^{*} j$, we have $\omega_{h}=\nu^{*} \omega_{j}$. Thus as elements of $H^{*}(M, \mathbb{C})$,

$$
\left[\omega_{h}\right]=\left[\nu^{*} \omega_{j}\right]=\nu^{*}\left[\omega_{j}\right]=\nu^{*}[\eta] .
$$

By Lemma $4,\left[\omega_{h}\right]$ is independent of the particular choice of $h$, and the proposition follows.

Corollary 2. For all $s>0$,

$$
\left\langle\operatorname{ch}_{\beta, s}\left(\mathcal{E}^{\omega}\right), \tau_{\eta}\right\rangle=\beta^{k / 2} /(k !) \int_{M} \widehat{A}(M) \wedge \mathrm{Ch}(V) \wedge \nu^{*}([\eta])
$$

Note. One can equally well pair $\operatorname{ch}_{\beta, s}\left(\mathcal{E}^{\omega}\right)$ with any element of $H C^{*}\left(\mathfrak{B}^{\omega}\right)$. Modulo growth conditions, there is a way of producing an element $\tau \in$ $H C^{k}\left(\mathfrak{B}^{\omega}\right)$ from a conjugacy class $\langle x\rangle$ of $\Gamma$ and a $k$-cocycle of the group $\Gamma_{x} /\{x\}$, where $\Gamma_{x}$ is the centralizer of $x$ in $\Gamma$ and $\{x\}$ is the subgroup generated by $x[\mathrm{Bu}]$. (The cocycle (62) comes from the special case when $\langle x\rangle=\langle e\rangle$.) However, the cyclic cohomology classes corresponding to $\langle x\rangle \neq$ $\langle e\rangle$ will pair with $\operatorname{ch}_{\beta, s}\left(\mathcal{E}^{\omega}\right)$ to give zero. The reason is that a cyclic $k$-cocycle $\tau$ based on $\langle x\rangle$ will have $\tau\left(g_{0}, \ldots, g_{k}\right)=0$ if $g_{0} g_{1} \ldots g_{k} \notin\langle x\rangle$. However, by the proof of Proposition 12, in the $s \rightarrow 0$ limit one sees that the terms with $g_{0} g_{1} \ldots g_{k} \neq e$ do not contribute to $\left\langle\operatorname{ch}_{\beta, s}\left(\mathcal{E}^{\omega}\right), \tau\right\rangle$.

\section{Reduction to the Index Bundle}

We first review some of the results of [MF]. Recall that $\Lambda$ is the reduced group $C^{*}$-algebra of $\Gamma$. Let $\mathcal{E}$ denote the $\mathbb{Z}_{2}$-graded $\Lambda$-bundle over $M$ given by $\mathcal{E}=\left(\widetilde{M} \times_{\Gamma} \Lambda\right) \otimes E$. The $L^{2}$-sections $\Gamma^{0}(\mathcal{E})$ of $\mathcal{E}$ form a right $\Lambda$-Hilbert module. The Dirac-type operator $\widetilde{D}$ is an odd densely-defined unbounded operator on $\Gamma^{0}(\mathcal{E})$. One can find finitely-generated right projective $\Lambda$-Hilbert submodules $F^{ \pm}$of $\Gamma^{0}\left(\mathcal{E}^{ \pm}\right)$and complementary $\Lambda$-Hilbert modules $G^{ \pm} \subset \Gamma^{0}\left(\mathcal{E}^{ \pm}\right)$such that $\widetilde{D}$ is diagonal with respect to the decomposition $\Gamma^{0}\left(\mathcal{E}^{ \pm}\right)=G^{ \pm} \oplus F^{ \pm}$, and writing $\widetilde{D}=\widetilde{D}_{G} \oplus \widetilde{D}_{F}$, in addition $\widetilde{D}_{G}: G^{ \pm} \rightarrow G^{\mp}$ is invertible. By definition, the index of $\widetilde{D}$ is

$$
\operatorname{Index}(\tilde{D}) \equiv\left[F^{+}\right]-\left[F^{-}\right] \in K_{0}(\Lambda) ;
$$

this is independent of the choice of $F^{ \pm}$. 
Now suppose that $\mathfrak{B}^{\infty}$ is a densely-defined subalgebra of $\Lambda$ which is stable with respect to the holomorphic functional calculus on $\Lambda$, and $\mathfrak{B}^{\omega} \subset$ $\mathfrak{B}^{\infty} \subset \Lambda$. A standard result in $K$-theory is that $K_{0}(\Lambda) \cong K_{0}\left(\mathfrak{B}^{\infty}\right)$ [Bo, Appendice]. There is a Chern character $\mathrm{Ch}_{\beta}$ from $K_{0}\left(\mathfrak{B}^{\infty}\right)$ to $\overline{H C_{*}\left(\mathfrak{B}^{\infty}\right)}$, the reduced cyclic homology of $\mathfrak{B}^{\infty}[\mathrm{Ka}]$. Let $\eta$ be a group $k$-cocycle on $\mathbb{C} \Gamma$ which extends to an element $\tau_{\eta}$ of the cyclic cohomology of $\mathfrak{B}^{\infty}$. By the explicit formula (62), $\tau_{\eta}$ is a reduced cyclic cohomology class if $k>0$.

We will sketch a proof of the following proposition. Many of the details are as in [Bi].

Proposition 15.

$$
\left\langle\mathrm{Ch}_{\beta}(\operatorname{Index}(\widetilde{D})), \tau_{\eta}\right\rangle=\beta^{k / 2} /(k !) \int_{M} \widehat{A}(M) \wedge \operatorname{Ch}(V) \wedge \nu^{*}([\eta]) .
$$

Proof: Define $\mathcal{E}^{\infty}$ to be $\left(\widetilde{M} \times_{\Gamma} \mathfrak{B}^{\infty}\right) \otimes E$. An examination of the proof of [MF] shows that $F^{ \pm}$and $G^{ \pm}$can be chosen to be of the form $F^{ \pm}=\mathcal{F}^{ \pm} \otimes_{\mathfrak{B} \infty}$ $\Lambda$ and $G^{ \pm}=\mathcal{G}^{ \pm} \otimes_{\mathfrak{B} \infty} \Lambda$, where $\mathcal{F}^{ \pm}$and $\mathcal{G}^{ \pm}$are subspaces of $\Gamma^{\infty}\left(\mathcal{E}^{\infty}\right)$. (This uses the fact that $\mathfrak{B}^{\infty}$ is stable with respect to the holomorphic functional calculus in $\Lambda$.) Write $\widetilde{D}_{\mathcal{F}^{ \pm}}$and $\widetilde{D}_{\mathcal{G}^{ \pm}}$for the restrictions of $\widetilde{D}$ to $\mathcal{F}^{ \pm}$and $\mathcal{G}^{ \pm}$ respectively. Put

$$
\mathcal{H}^{ \pm}=\mathcal{G}^{ \pm} \oplus \mathcal{F}^{ \pm} \oplus \mathcal{F}^{\mp}
$$

For $\alpha \in \mathbb{C}$, define $R_{\alpha}^{ \pm}: \mathcal{H}^{ \pm} \rightarrow \mathcal{H}^{\mp}$ by

$$
R_{\alpha}^{ \pm}=\left(\begin{array}{ccc}
\tilde{D}_{\mathcal{G}^{ \pm}} & 0 & 0 \\
0 & \widetilde{D}_{\mathcal{F}^{ \pm}} & \alpha \\
0 & \alpha & 0
\end{array}\right)
$$

We have that $\widetilde{D}_{\mathcal{G}^{ \pm}}$is invertible. Put

$$
S_{\alpha}^{ \pm}=\left(\begin{array}{cc}
\tilde{D}_{\mathcal{F}^{ \pm}} & \alpha \\
\alpha & 0
\end{array}\right)
$$

and let

$$
S_{\alpha}^{ \pm} \otimes_{\mathfrak{B} \infty} \Lambda: F^{ \pm} \oplus F^{\mp} \rightarrow F^{\mp} \oplus F^{ \pm}
$$

be the extension to a bounded operator on finitely-generated Hilbert $\Lambda$ modules. As $\widetilde{D}_{F}$ is a bounded operator, it follows that $S_{\alpha}^{ \pm} \otimes_{\mathfrak{B} \infty} \Lambda$ is invertible for $\alpha$ large. Then the fact that $\mathfrak{B}^{\infty}$ is stable under the holomorphic 
functional calculus in $\Lambda$ implies that $S_{\alpha}^{ \pm}$is also invertible for $\alpha$ large. Thus $R_{\alpha}^{ \pm}$is invertible for $\alpha$ large. We define $\exp \left(-\mathrm{TR}_{\alpha}^{2}\right)$ by the Duhamel expansion in $\alpha$. As $R_{\alpha}$ differs from $\widetilde{D} \oplus 0$ by a finite-rank operator in the sense of [Kas], there is no problem in showing that $\exp \left(-\mathrm{TR}_{\alpha}^{2}\right)$ is well-defined.

Extend the $\mathfrak{B}^{\omega}$-connection $\nabla$ on $\mathcal{E}^{\omega}$ to a $\mathfrak{B}^{\infty}$-connection on

$$
\mathcal{E}^{\infty}=\mathcal{E}^{\omega} \otimes_{\mathfrak{B}^{\omega}} \mathfrak{B}^{\infty}
$$

Let $\nabla_{\mathcal{F}}$ be a $\mathfrak{B}^{\infty}$-connection on $\mathcal{F}$ and let

$$
\nabla^{\prime}=\nabla \oplus \nabla_{\mathcal{F}}
$$

be the sum connection on $\mathcal{H}$. Define the Chern character

$$
\operatorname{ch}_{\beta, s, \alpha}(\mathcal{H})=\operatorname{STR} \exp \left(-\beta\left(\nabla^{\prime}+s R_{\alpha}\right)^{2}\right) \in \overline{\widehat{\Omega}}\left(\mathfrak{B}^{\infty}\right)
$$

by a Duhamel expansion in $\nabla^{\prime}$. For $\alpha=0$, we have

$$
\operatorname{ch}_{\beta, s, 0}(\mathcal{H})=\operatorname{ch}_{\beta, s}\left(\mathcal{E}^{\infty}\right)-\operatorname{STR} \exp \left(-\beta \nabla_{\mathcal{F}}^{2}\right)
$$

Now $\operatorname{STR} \exp \left(-\beta \nabla_{\mathcal{F}}^{2}\right) \in \overline{\widehat{\Omega}}\left(\mathfrak{B}^{\infty}\right)$ represents $\mathrm{Ch}_{\beta}([\mathcal{F}])[\mathrm{Ka}]$. If we can show that $\operatorname{ch}_{\beta, s, 0}(\mathcal{H})$ is zero in $\bar{H}_{*}\left(\mathfrak{B}^{\infty}\right)$ then we will have that as classes in $\bar{H}_{*}\left(\mathfrak{B}^{\infty}\right)$,

$$
\operatorname{ch}_{\beta, s}\left(\mathcal{E}^{\infty}\right)=\operatorname{STR} \exp \left(-\beta \nabla_{\mathcal{F}}^{2}\right)=\mathrm{Ch}_{\beta}([\mathcal{F}])=\operatorname{Ch}_{\beta}(\operatorname{Index}(\widetilde{D}))
$$

and the proposition will follow.

A standard homotopy argument shows that the class of $\operatorname{ch}_{\beta, s, \alpha}(\mathcal{H})$ in $\bar{H}_{*}\left(\mathfrak{B}^{\infty}\right)$ is independent of $\alpha$. Take $\alpha$ large enough that $R_{\alpha}$ is invertible.

We define a pseudodifferential calculus as in $[\mathrm{MF}]$, except that the symbol $\sigma(m, \xi)$ will take value in End ${ }_{\mathfrak{B} \infty}\left(\mathcal{E}_{m}^{\infty}\right)$. Then $R_{\alpha}$ is an elliptic firstorder $\psi$ do. (In terms of the "fibration" picture, it corresponds to a smooth family of elliptic first-order vertical $\psi$ do's.) As in the usual calculus of $\psi$ do's, $R_{\alpha}$ has a parametrix $P_{\alpha}$, an order $-1 \psi$ do, such that

$$
I-R_{\alpha} P_{\alpha}=K_{1 \alpha} \text { and } I-P_{\alpha} R_{\alpha}=K_{2 \alpha}
$$

where $K_{1 \alpha}$ and $K_{2 \alpha}$ are smoothing operators. It follows that

$$
\left(R_{\alpha}\right)^{-1}=P_{\alpha}+K_{2 \alpha}\left(R_{\alpha}\right)^{-1}
$$


is also an order $-1 \psi$ do.

Define a connection $\nabla_{\mathcal{H}^{-}}^{\prime \prime}$ on $\mathcal{H}^{-}$by

$$
\nabla_{\mathcal{H}^{-}}^{\prime \prime}=\left(R_{\alpha}^{-}\right)^{-1} \nabla_{\mathcal{H}^{+}}^{\prime} R_{\alpha}^{-}
$$

and define $\nabla^{\prime \prime}$ to be $\nabla_{\mathcal{H}^{+}}^{\prime} \oplus \nabla_{\mathcal{H}^{-}}^{\prime \prime}$ Then

$$
\nabla_{\mathcal{H}^{+}}^{\prime \prime}-\nabla_{\mathcal{H}^{+}}^{\prime}=0
$$

and

$$
\nabla_{\mathcal{H}^{-}}^{\prime \prime}-\nabla_{\mathcal{H}^{-}}^{\prime}=\left(R_{\alpha}^{-}\right)^{-1}\left(\nabla_{\mathcal{H}^{+}}^{\prime} R_{\alpha}^{-}-R_{\alpha}^{-} \nabla_{\mathcal{H}^{+}}^{\prime}\right)
$$

is an order -1 operator. We have a homotopy of connections on $H$ from $\nabla^{\prime}$ to $\nabla^{\prime \prime}$ given by $\nabla^{\prime}+u\left(\nabla^{\prime \prime}-\nabla^{\prime}\right), u \in[0,1]$. It follows as in [Bi, Prop. 2.10] that $\operatorname{ch}_{\beta, s, \alpha}(H)=\mathrm{STR} \exp \left(-\beta\left(\nabla^{\prime}+s R_{\alpha}\right)^{2}\right)$ represents the same class in $\bar{H}_{*}\left(\mathfrak{B}^{\infty}\right)$ as $\operatorname{STR} \exp \left(-\beta\left(\nabla^{\prime \prime}+s R_{\alpha}\right)^{2}\right)$.

We claim that if STR $\exp \left(-\beta\left(\nabla^{\prime \prime}+s R_{\alpha}\right)^{2}\right)$ is expanded in $\nabla^{\prime \prime}$, the terms vanish algebraically. To see this formally, write $\nabla^{\prime \prime}+s R_{\alpha}$ in terms of the decomposition $\mathcal{H}=\mathcal{H}^{+} \oplus \mathcal{H}^{-}$as

$$
\begin{aligned}
& \nabla^{\prime \prime}+s R_{\alpha}=\left(\begin{array}{cc}
\nabla_{\mathcal{H}^{+}}^{\prime} & s R_{\alpha}^{-} \\
s R_{\alpha}^{+} & \left(R_{\alpha}^{-}\right)^{-1} \nabla_{\mathcal{H}^{+}}^{\prime} R_{\alpha}^{-}
\end{array}\right)= \\
& \left(\begin{array}{cc}
I & 0 \\
0 & s^{-1}\left(R_{\alpha}^{-}\right)^{-1}
\end{array}\right)\left(\begin{array}{cc}
\nabla_{\mathcal{H}^{+}}^{\prime} & I \\
s^{2} R_{\alpha}^{-} R_{\alpha}^{+} & \nabla_{\mathcal{H}^{+}}^{\prime}
\end{array}\right)\left(\begin{array}{cc}
I & 0 \\
0 & s\left(R_{\alpha}^{-}\right)
\end{array}\right)
\end{aligned}
$$

and so formally,

$$
\begin{gathered}
\operatorname{STR} \exp \left(-\beta\left(\nabla^{\prime \prime}+s R_{\alpha}\right)^{2}\right)=\operatorname{STR} \exp \left(-\beta\left(\begin{array}{cc}
\nabla_{\mathcal{H}^{+}}^{\prime} & I \\
s^{2} R_{\alpha}^{-} R_{\alpha}^{+} & \nabla_{\mathcal{H}^{+}}^{\prime}
\end{array}\right)^{2}\right) \\
\in \overline{\widehat{\Omega}}_{*}\left(\mathfrak{B}^{\infty}\right) .
\end{gathered}
$$

However, expanding (119) in $\nabla_{\mathcal{H}^{+}}^{\prime}$, one finds that (119) vanishes for algebraic reasons.

(To see this last point, consider an analogous statement in the finitedimensional case. For $A, B \in M_{N}(\mathbb{C})$ put

$$
\mathrm{M}=\left(\begin{array}{cc}
A & I \\
B & A
\end{array}\right) \in M_{2 N}(\mathbb{C})
$$


Then $\operatorname{det}(\mathrm{M})=\operatorname{det}\left(A^{2}-B\right)$ and if $A^{2}-B$ is invertible,

$$
\mathrm{M}^{-1}=\left(\begin{array}{cc}
\left(A^{2}-B\right)^{-1} A & -\left(A^{2}-B\right)^{-1} \\
I-A\left(A^{2}-B\right)^{-1} A & A\left(A^{2}-B\right)^{-1}
\end{array}\right)
$$

Thus $\operatorname{Str} \mathrm{M}^{-1}=0$. If $\lambda \notin \operatorname{Spec}(\mathrm{M})$, by changing $\mathrm{A}$ to $A-\lambda I$, we obtain that $\operatorname{Str}(\mathrm{M}-\lambda I)^{-1}=0$. Then by the functional calculus, if $f$ is a holomorphic function in a neighborhood of $\operatorname{Spec}(M), \operatorname{Str} f(M)=0$.)

This formal argument can be made rigorous as in [Bi, Prop. 2.17].

Note. If $M$ is odd-dimensional then one can use Quillen's formalism [Q] to define the odd Chern character

$$
\operatorname{ch}_{\beta, s}\left(\mathcal{E}^{\infty}\right)=\operatorname{Tr}_{\sigma} \exp \left(-\beta(\nabla+s Q \sigma)^{2}\right) \in \overline{\widehat{\Omega}}_{o d d}\left(\mathfrak{B}^{\infty}\right)
$$

The operator $\widetilde{D}$ gives an element $\operatorname{Index}(\widetilde{D})$ of $K_{1}\left(\mathfrak{B}^{\infty}\right)$ [Kas]. Using a suspension argument as in $[B F]$, one can show that Proposition 15 also holds in the odd case.

CoRollary 3 [CM]. If $\Gamma$ is a hyperbolic group in the sense of Gromov $[\mathrm{GH}]$ then for all $[\eta] \in H^{*}(\Gamma ; \mathbb{C})$, the higher-signature $\int_{M} L(M) \wedge \nu^{*}([\eta])$ is an (orientation-preserving) homotopy invariant of $M$.

Proof: Let $\mathfrak{B}^{\infty}$ be the algebra

$$
\mathfrak{B}^{\infty}=\left\{A \in \Lambda: \tilde{\partial}^{k}(A) \text { is bounded for all } k \in \mathbf{N}\right\}
$$

where $\widetilde{\partial}$ is the operator of [CM, p. 383]. By [CM, p. 385], if $[\eta] \in H^{*}(\Gamma ; \mathbb{C})$ then $[\eta]$ can be represented by a group cocycle $\eta$ such that $\tau_{\eta}$ extends to a cyclic cocycle on $\mathfrak{B}^{\infty}$. Letting $\widetilde{D}$ be the signature operator, the result of Mishchenko and Kasparov [Mi, Kas, HS] on the homotopy invariance of

$$
\operatorname{Index}(\widetilde{D}) \in K_{0}(\Lambda) \cong K_{0}\left(\mathfrak{B}^{\infty}\right)
$$

along with Corollary 2 implies the result. (As usual when dealing with the signature operator, it is irrelevant whether or not $M$ is spin.) 


\section{Bivariant Extension}

Let $\mathfrak{A}$ be the $C^{*}$-algebra $C(M)$. Then $\left(\Gamma^{0}(\mathcal{E}), \widetilde{D}\right)$ forms an unbounded $(\mathfrak{A}, \Lambda)$ Kasparov module, and so gives an element of $K K(\mathfrak{A}, \Lambda)$ [BJ]. A bivariant Chern character $\mathrm{ch}_{\beta, s}$ was defined in [Lo1] in the case of finitedimensional projective modules, and it was indicated that the bivariant Chern character should be well-defined whenever there is a good notion of trace on the Hilbert modules. Such is the case here. The bivariant Chern character is a combination of Quillen's superconnection Chern character $[Q]$ and the entire cyclic cocycle of [JLO]. In the setup of Section IV, given $\eta \in Z^{k}(\Gamma ; \mathbf{C})$ such that $\tau_{\eta}$ pairs with $\mathfrak{B}^{\infty}$, there is a corresponding entire cyclic cocycle $\left\langle\operatorname{ch}_{\beta, s}, \tau_{\eta}\right\rangle \in C_{\epsilon}^{*}\left(C^{\infty}(M)\right)$. It is given explicitly as follows:

Definition: For $a_{0}, \ldots, a_{m} \in C^{\infty}(M)$,

$$
\begin{aligned}
& \left\langle\operatorname{ch}_{\beta, s}, \tau_{\eta}\right\rangle\left(a_{0}, \ldots, a_{m}\right)=\beta^{-m / 2}\left\langle\int_{0}^{\beta} \ldots \int_{0}^{u_{m-1}} \operatorname{STR} a_{0} \exp \left(-u_{1} D_{s}^{2}\right)\right. \\
& {\left[D_{s}, a_{1}\right] \exp \left(-u_{2} D_{s}^{2}\right)\left[D_{s}, a_{2}\right] \ldots\left[D_{s}, a_{m}\right] \exp \left(-\left(\beta-u_{1}-\ldots-u_{m}\right) D_{s}^{2}\right)} \\
& \left.d u_{m} \ldots d u_{1}, \tau_{\eta}\right\rangle .
\end{aligned}
$$

(Note that the $\left\langle\operatorname{ch}_{\beta, s}, \tau_{\eta}\right\rangle(1)$ of equation (125) equals the $\left\langle\operatorname{ch}_{\beta, s}, \tau_{\eta}\right\rangle$ of Proposition 12.)

As before, the class of $\left\langle\operatorname{ch}_{\beta, s}, \tau_{\eta}\right\rangle$ in $H_{\epsilon}^{*}\left(C^{\infty}(M)\right)$ is independent of $s$. As in Section V, we can take the $s \rightarrow 0$ limit to obtain that $\left\langle\operatorname{ch}_{\beta, s}, \tau_{\eta}\right\rangle$ is cohomologous to the entire cyclic cocycle $\left\langle\operatorname{ch}_{\beta, 0}, \tau_{\eta}\right\rangle$ given by

$$
\begin{aligned}
\left\langle\operatorname{ch}_{\beta, 0}, \tau_{\eta}\right\rangle\left(a_{0}, \ldots, a_{m}\right)= & \beta^{k / 2} /(k ! m !) \int_{M} \widehat{A}(M) \wedge \operatorname{Ch}(V) \wedge \\
& \omega \wedge a_{0} d a_{1} \wedge d a_{2} \wedge \ldots \wedge d \dot{a}_{m} .
\end{aligned}
$$

Here $\omega$ is the differential form of (65).

If $W \in K^{0}(M)$ is represented by a projection $p \in M_{r}\left(C^{\infty}(M)\right)$, let $\mathrm{Ch}_{*}(p)$ be the entire cyclic cycle of [GS]. Then we obtain that $\left\langle\operatorname{ch}_{\beta, s}, \tau_{\eta}\right\rangle\left(\mathrm{Ch}_{*}(p)\right)$ is proportionate to $\int_{M} \widehat{A}(M) \wedge \mathrm{Ch}(V) \wedge \omega \wedge \mathrm{Ch}(W)$. Note that in the case of the signature operator, the entire cyclic cohomology class of $\left\langle\operatorname{ch}_{\beta, s}, \tau_{\eta}\right\rangle$ is not a homotopy invariant, as otherwise one could take $[\eta]$ to be a 0 -group cocycle and conclude that the rational $L$-class is a homotopy invariant, which is false. 


\section{References}

[AS1] M. Atiyah, I. Singer, The index of elliptic operators: III, Ann. Math. 87 (1968), 546-604.

[AS2] M. Atiyah, I. Singer, The index of elliptic operators: IV, Ann. Math. 93 (1971), 119-138.

[At] M. ATIYAH, Elliptic operators, discrete groups and von Neumann algebras, Astérisque 32/33 (1976), 43-72.

[Bi] J.-M. BismuT, The Atiyah-Singer index theorem for families of Dirac operators: two heat equation proofs, Inv. Math. 83 (1986), 91-151.

[BF] J.-M. Bismut, D. Freed, The analysis of elliptic families II, Comm. Math. Phys. 107 (1986), 103-163.

[BJ] S. BAAJ, P. JULG, Théorie bivariante de Kasparov et opérateurs non bornés dans les $C^{*}$-modules Hilbertiens, C.R. Acad. Sci. Paris 296, Ser. I (1983), 875-878.

[Bo] J.-B. Bost, Principe d'Oka, K-théorie et systèmes dynamiques noncommutatifs, Inv. Math. 101 (1990), 261; and references therein.

[Br] K. Brown, Cohomology of Groups, Springer-Verlag, New York (1982).

[Bu] D. Burghelea, The cyclic homology of group rings, Comm. Math. Helv. 60 (1985), 354-365.

[CGT] J. Cheeger, M. Gromov, M. Taylor, Finite propagation speed, kernel estimates for functions of the Laplace operator and the geometry of complete Riemannian manifolds, J. Diff. Geom. 17 (1982), 15-53.

[CM] A. Connes, H. Moscovici, Cyclic cohomology, the Novikov conjecture and hyperbolic groups, Topology 29 (1990), 345-388.

[Co1] A. Connes, Noncommutative differential geometry", Publ. Math. IHES 62 (1985), 41-144.

[Co2] A. Connes, Entire cyclic cohomology of Banach algebras and characters of $\theta$-summable Fredholm modules, $K$-Theory 1 (1988), 519-548.

[Co3] A. Connes, Cyclic cohomology and noncommutative differential geometry, Proc. ICM 1986 at Berkeley, AMS (1987), 879-889; and references therein.

[G] E. Getzler, Pseudodifferential operators on supermanifolds and the Atiyah Singer index theorem, Comm. Math. Phys. 92 (1983), 163-178.

[GH] E. Ghys, P. DE LA HARPE, EDs., Sur les Groupes Hyperboliques D'après Mikhael Gromov, Birkhauser, Boston (1990).

[GM] P. Griffiths, J. Morgan, Rational Homotopy Theory and Differential Forms, Birkhauser, Boston (1981).

[GS] E. Getzler, A. Szenes, On the Chern character of a theta-summable Fredholm module, J. Func. Anal. 84 (1989), 343.

[Hi] N. Higson, A primer on KK-theory, in "Operator Theory, Operator Algebras and Applications", Proc. Symp. Pure Math. 51, AMS (1980), 239-283.

[HS] M. Hilsum, G. Skandalis, Invariance par homotopie de la signature à coefficients dans un fibré presque plat, preprint, Collège de France (1980).

[JLO] A. JAFFe, A. Lesniewski, K. Osterwalder, Quantum K-theory. I. The Chern character, Comm. Math. Phys. 118 (1988), 1-14.

[Ka] M. Karoubi, Homologie Cyclique et $K$-théorie, Astérisque 149 (1987).

[Kas] G. KASPARov, Operator $K$-theory and its applications: elliptic operators, group representations, higher signatures, $C^{*}$-extensions, Proc. ICM 1983 
at Warsaw, North-Holland, Amsterdam (1984), 987-1000; and references therein

G. KasParov, Equivariant KK-theory and the Novikov conjecture, Inv. Math. 91 (1988), 147-201.

[Lo1] J. LoTT, Superconnections and noncommutative de Rham homology, preprint (1990).

[Lo2] J. LotT, Heat kernels on covering spaces and topological invariants, to appear, J. Diff. Geom.

[Lu] G. LusztiG, Novikov's higher signature and families of elliptic operators, J. Diff. Geom. 7 (1971), 229-256.

[Mal] A. Maluios, Topological algebras - selected topics, North-Holland, Amsterdam (1986).

[Mi] A. MishCHENKo, $C^{*}$-algebras and $K$-theory, Springer LNM 763 (1974), 262274.

[MF] A. Mishchenko, A. Fomenko, The index of elliptic operators over $C^{*}$-algebras, Izv. Akad. Nauk SSSR, Ser. Mat. 43 (1979), 831-859.

[Mo] H. MoscovicI, Cyclic cohomology and invariants of multiply-connected manifolds, Proc. ICM 1990 at Kyoto, to appear; and references therein.

[No] S. Novikov, Pontryagin classes, the fundamental group and some problems of stable algebras, in " Essays on Topology and Related Topics, Mémoires Dédiés à Georges de Rham", Springer, Berlin (1969), 147-155.

[Qu] D. Quillen, Superconnections and the Chern character, Topology 24 (1985), 89-85.

[Ro] J. Rosenberg, $C^{*}$-algebras, positive scalar curvature and the Novikov conjecture, Publ. Math. IHES 58 (1983), 197-212.

[Te] N. Teleman, The index of signature operators on Lipschitz manifolds, Publ. Math. IHES 58 (1983), 39-78.

John Lott

Department of Mathematics

University of Michigan

Ann Arbor, MI 48109

e-mail: lott@math.lsa.umich.edu

Submitted: October 1991 